\title{
Amour, imagination et poésie dans l'œuvre médicale de Gentile da Foligno
}

Gentile da Foligno est une figure bien connue des historiens de la médecine ${ }^{1}$. Mort de la peste noire en 1348 après avoir lui-même rédigé un Consilium de pestilentia, celui que l'on nomma, à l'instar d'Avicenne, le prince des médecins (medicorum princeps), parfois plus modestement le speculator, laissa derrière lui une œuvre médicale monumentale, restée largement inédite. C'est toutefois son commentaire au Canon d'Avicenne, le plus ample du XIV ${ }^{\mathrm{e}}$ siècle, qui lui assura rapidement une renommée dans toute la Péninsule et au-delà, dans le reste de l'Europe. Cet immense travail exégétique, touchant aussi bien à la théorie qu'à la pratique de la médecine, fut fréquemment copié, puis édité à plusieurs reprises à la Renaissance, et continua d'être utilisé dans l'enseignement universitaire jusqu'au XVII ${ }^{\mathrm{e}}$ siècle ${ }^{2}$. Il faut dire que son auteur ne manque jamais de situer son propos par rapport aux débats antérieurs, citant pêle-mêle ses illustres prédécesseurs Taddeo Alderotti, Antoine de Parme, Dino del Garbo, Pietro Torrigiano ou encore Pietro d'Abano, ce qui rendait l'ouvrage fort utile pour l'enseignement. Pour autant, le caractère encyclopédique et exhaustif de ce commentaire ne saurait constituer l'unique raison d'une telle longévité. Un tel succès repose en outre sur l'originalité de certains de ses propos.

Les remarques précédentes valent plus particulièrement pour le thème qui nous occupe ici, à savoir les rapports entre le corps et l'esprit dans la littérature, la philosophie et la médecine. Comme l'on sait, de nombreux passages du Canon d'Avicenne, notamment dans les premières fen du livre I, traitent des diverses fonctions de l'âme et de leur rapport avec le corps. L'analyse des lectures médiévales de ces chapitres, la plupart du temps négligées par les historiens de la philosophie, présenterait donc un intérêt historique et philosophique en soi. Ce n'est pourtant pas, ou pas seulement, pour cette raison que le commentaire de Gentile

1 Pour une vue d'ensemble sur le personnage et son œuvre, voir J. Chandelier, Avicenne et la médecine en Italie. Le Canon dans les universités (1200-1350), Paris 2017, partie I, chapitre 3. On consultera aussi M. L. Ceccarelli Lemut, «Gentile da Foligno », dans Dizionario biografico degli Italiani, vol. 52, Rome 1999, 162-167 et R. French, Canonical Medicine. Gentile da Foligno and Scholasticism, Leiden-Boston-Köln 2001.

2 Sur la fortune de ce commentaire, cf. N. Siraisi, Avicenna in Renaissance Italy. The Canon and Medical Teaching in Italian Universities after 1500, Princeton (N. J.) 1987, en partic. 131.

Aurélien Robert, CNRS, Centre d’Études Supérieures de la Renaissance

Ә Open Access. (C) 2020 Aurélien Robert, published by De Gruyter. (๕)BY-NC-ND This work is licensed under a Creative Commons Attribution-NonCommercial-NoDerivatives 4.0 International License.

https://doi.org/10.1515/9783110615937-010 
da Foligno constitue un témoignage de grande valeur. Il est aussi précieux parce qu'il offre une illustration parfaite de l'univers culturel tout à fait singulier dans lequel s'épanouissait une génération d'intellectuels italiens, médecins, notaires, astrologues ou juristes, à l'ombre des grands noms de Cavalcanti, Dante, Boccace ou Pétrarque ${ }^{3}$.

En effet, loin de se cantonner à la discipline qu'ils enseignaient dans quelque université du nord de l'Italie, nombre de médecins pratiquaient l'ensemble des savoirs de leur temps : la philosophie, bien sûr, mais aussi la poésie ${ }^{4}$. Certains d'entre eux se détachent nettement dans ce paysage intellectuel, comme Dino del Garbo, célèbre pour son commentaire médical et philosophique à la chanson Donna me prega de Guido Cavalcanti, qui restera une référence fondamentale dans les débats sur l'amour jusqu'au XVI ${ }^{\mathrm{e}}$ siècle ${ }^{5}$. Moins connu, quoique tout aussi intéressant, le médecin florentin Pietro Torrigiano, auteur d'un influent commentaire au Tegni de Galien, et qui rédigea des sonnets en langue vernaculaire ${ }^{6}$. Ces poèmes montrent un fort attachement à la philosophie, mais aussi à la théologie. D’ailleurs, certaines chroniques affirment qu'il préféra abandonner l'enseignement de la médecine pour entrer dans les ordres à la fin de sa vie ${ }^{7}$. Il faudrait certainement ajouter Gentile da Foligno à ce panthéon. Il connaissait bien ces deux personnages et était conscient, comme eux, de la grande porosité entre discours médical, philosophique, théologique et poétique. Aussi n'hésitait-il jamais à préciser tel concept philosophique, lorsque les philosophes eux-mêmes avaient laissé dans l'ombre un point de doctrine ${ }^{8}$. Il ne daignait pas non plus offrir ses

3 Sur ce contexte intellectuel, voir E. Coccia et S. Piron, « Poésie, sciences et politique. Une génération d'intellectuels italiens (1290-1330) », Revue de synthèse 129 (2008), $6^{\text {ème }}$ série, n³, 1-38. 4 Qu'il nous soit permis de renvoyer ici au volume que nous avons co-édité sur ce thème : J. Chandelier et A. Robert (éds.), Frontières des savoirs en Italie à l'époque des premières universités (XIII ${ }^{e}-X V^{e}$ siècle), Rome 2015.

5 Cf. E. Fenzi, La Canzone d'amore di Guido Cavalcanti e i suoi antichi commenti, Gênes 1999. À propos de l'influence de ce commentaire, voir M.-M. Fontaine, « La lignée des commentaires à la chanson de Guido Cavalcanti Donna me prega : évolution des relations entre philosophie, médecine et littérature dans le débat sur la nature d'Amour (de la fin du XIII ${ }^{\mathrm{e}}$ siècle à celle du XVI ${ }^{\mathrm{e}}$ siècle) », dans J. Céard (éd.), La folie et le corps, Paris 1985, 159-178. Pour des renseignements sur la carrière et l'œuvre de Dino del Garbo, cf. N. Siraisi, Taddeo Alderotti and his Pupils, Princeton 1981, 55-64.

6 Nous connaissons de lui sept sonnets, édités par Sergio Lubello sous la rubrique Maestro Torrigiano, dans I poeti della scuola siciliana, vol. 3, Milan 2008, 415-438.

7 Certaines sources disent qu'il est entré chez les Dominicains, d'autres affirment qu'il était Chartreux. Cf. Siraisi, Taddeo Alderotti, 65-66.

8 Voir par exemple J. Chandelier, « Expérience, expérimentation et connaissance dans la médecine scolastique italienne du $14^{\mathrm{e}}$ siècle », dans T. Bénatouil et I. Draelants (éds.), Expertus sum. L'expérience par les sens dans la philosophie naturelle médiévale, Florence 2011, 385-403 ; 
services à son ami Cino da Pistoia, juriste et néanmoins grand poète du dolce stil novo, lorsqu'il faisait appel lui lors de quelque procès difficile ${ }^{9}$. Plus intéressant pour notre présent propos, Gentile composa lui aussi des poèmes en langue vernaculaire, malheureusement perdus aujourd'hui. Ces quelques éléments nous invitent à l'intégrer de plein droit dans le milieu intellectuel rapidement décrit ci-dessus.

À défaut de pouvoir proposer une étude globale des œuvres de Gentile da Foligno, tâche impossible dans l'espace qui nous est offert ici, nous concentrerons nos efforts sur son analyse de la passion amoureuse, thème qui mobilise à la fois la médecine, la philosophie et la littérature. Alors que la mélancolie amoureuse avait déjà fait l'objet d'un débat littéraire particulièrement intense en Italie, dont l'acmé peut être située dans les échanges poétiques entre Dante et Guido Cavalcanti, alors qu'une importante production médicale sur ce même sujet avait vu le jour en Occident, dès le XII ${ }^{\mathrm{e}}$ siècle ${ }^{10}$, que peut nous apporter le témoignage tardif de Gentile da Foligno ? Ses réflexions sur l'amour, jamais étudiées de près à ce jour, laissent transparaître une pensée singulière, tant sur les rapports entre l'âme et le corps, que sur la nécessaire complémentarité entre le point de vue du médecin et celui du poète. Elles indiquent en outre un changement historique et philosophique important et durable des conceptions poético-médicales de l'amour. Quelle est l'origine de la passion amoureuse ? Comment la pensée et l'imagination agissent-elles sur le corps ? Quel pouvoir doit-on attribuer à la parole dans les manifestations physiques qui accompagnent la passion ? Autant de questions qui sont intimement liées dans les débats médiévaux sur l'amour, sans être nécessairement traitées ensemble ${ }^{11}$. Gentile da Foligno les aborde de

J. Chandelier et A. Robert, « Nature humaine et complexion du corps chez les médecins italiens de la fin du Moyen Âge », Revue de synthèse 134 (2013), $6^{\mathrm{e}}$ série, nº4, 473-510.

9 H. U. Kantorowicz, "Cino da Pistoia ed il primo trattato di medicina legale », Archivio storico italiano, ser. 5, t. 37, 1906, 115-128. Gentile da Foligno rédigea en effet un consilium en réponse à la demande expresse de Cino da Pistoia, son ami et collègue à Pérouse, à propos de la possibilité d'une naissance au huitième mois de grossesse. Un mari jaloux contestait la paternité de l'enfant auquel sa femme allait donner naissance. Gentile da Foligno explique donc à son collègue pérugin comment la médecine analysait la possibilité d'un accouchement au huitième mois qui était au cœur de la discorde conjugale. Sur le contexte et le sens qu'il faut donner à ce texte de Gentile da Foligno, cf. J. Chandelier et M. Nicoud, « Entre droit et médecine. Les origines de la médecine légale en Italie (XIII $-\mathrm{XIV}^{\mathrm{e}}$ siècles) », dans Chandelier et Robert (éds.), Frontières des savoirs en Italie, 233-293.

10 Cette littérature a déjà fait l'objet de nombreuses études. Mentionnons, par exemple, M. Ciavolella, La malattia d'amore dall'Antichità al Medioevo, Rome 1976 et M. F. Wack, Lovesickness in the Middle Ages. The Viaticum and its Commentaries, Philadelphia 1990.

11 Nous avons déjà abordé cette question dans A. Robert, « Le pouvoir des incantations selon les médecins du Moyen Âge (XIII ${ }^{\mathrm{e}}-\mathrm{XV}^{\mathrm{e}}$ siècle) », dans N. Bériou, J. -P. Boudet et I. Rosier-Catach 
concert dans son commentaire au Canon d'Avicenne. Les causes et les effets de cette passion, ainsi que ses remèdes, seraient noués dans le jeu subtil des pouvoirs de l'imagination et de la parole. Or, si l'importance de l'imagination, dans ce contexte, a été remarquée de longue date, et même théorisée par Giorgio Agamben $^{12}$, la place de la parole, en revanche, et en particulier de la parole poétique, constitue un thème plus rare, en tout cas chez les médecins.

Nous tenterons donc de montrer le caractère exceptionnel de ce témoignage. Il s'agira surtout de préciser les raisons de cette singularité, en insistant sur quelques caractéristiques saillantes du commentaire de Gentile da Foligno au chapitre du Canon d'Avicenne consacré à l'amour : l'influence de Platon et du platonisme, les réflexions originales sur les rapports entre poésie et médecine, ou encore la reprise d'une discussion de Pietro d'Abano sur l'efficacité des pratiques incantatoires, appliquée de manière inédite au thème de l'amour.

\section{Avicenne, l'amour et les rapports entre l'âme et le corps}

Les travaux de Jackie Pigeaud ont montré que dès l'Antiquité l'analyse des « maladies de l'âme » en général, et de la mélancolie amoureuse en particulier, a constitué un champ d'expérimentation pour les médecins et les philosophes, traçant des lignes de partage, parfois très nettes, entre différentes conceptions des rapports entre l'âme et le corps ${ }^{13}$. S'opposaient alors diverses formes de dualisme et de monisme, les uns conférant un primat au corps, les autres préférant insister sur le rôle de l'âme elle-même. Galien, par exemple, dans son commentaire aux Pronostics d'Hippocrate, traduit en latin au $\mathrm{XI}^{\mathrm{e}}$ siècle à partir d'une version arabe, range l'amour maladif aux côtés d'autres émotions, comme la tristesse ou la joie par exemple, dont l'origine se trouve à la fois dans la matière et la pensée ${ }^{14}$. Autrement dit, pour Galien, l'amour devenu pathologique ne serait pas véritablement une maladie à part entière, mais serait plutôt une forme de dérèglement émotionnel, venant perturber le fonctionnement du corps, et qui proviendrait in fine d'un déséquilibre dans la complexion du corps.

(éds.), Le pouvoir des mots au Moyen Âge, Turnhout 2014, 459-489.

12 G. Agamben, Stanze. La parole et le fantasme dans la culture occidentale, Paris 1998.

$13 \mathrm{~J}$. Pigeaud, La Maladie de l'âme. Étude sur la relation de l'âme et du corps dans la tradition médico-philosophique antique, Paris 2006.

14 Galien, In Hippocratis Prognosticorum commentaria, éd. I. Heeg, Corpus Medicorum Graecorum V, 9, 2, Leipzig-Berlin 1915, 206-207. 
En Occident, le point de vue médical sur l'amour a sensiblement évolué à partir du XII ${ }^{\mathrm{e}}$ siècle, avec l'arrivée de nouvelles traductions de textes de langue arabe et le développement de l'enseignement médical, notamment à Salerne, puis dans les universités du nord de l'Italie. Ainsi, le Viaticum peregrinantis, un guide de santé pour les voyageurs attribué à Constantin l'Africain-mais qui est en fait une adaptation latine du Kitāb Zād al-musāfir du médecin Ibn al-Jazzār (mort en 979) ${ }^{15}$-est rapidement devenu l'une des sources les plus souvent citées à propos de l'amour $^{16}$. Avec ce traité, la passion amoureuse devient une maladie distincte. La mélancolie amoureuse y est définie comme une maladie qui touche le cerveau (est cerebro contiguus) et qui est intimement liée au désir et au plaisir.

Il s'agit en effet d'un intense désir, accompagné de concupiscence excessive et d'une affection des pensées. C’est pourquoi certains philosophes disent : eros est le nom désignant le plus grand plaisir. De même, en effet, que la fidélité est la forme ultime de l'amour, de même eros est la forme extrême du plaisir ${ }^{17}$.

Selon l'auteur, il est difficile de déterminer si la cause se situe dans le corps ou dans l'âme. Il s'agit parfois d'un simple excès d'humeur, auquel cas seul le coït permettra de réguler le corps. S'il s'agit en revanche d'un dérèglement du désir et des pensées, sans autre cause que la vue ou l'imagination d'une « belle forme », alors il faudra distraire l'âme.

À l'évidence, l'auteur hésite ici entre un modèle platonicien, centré sur l'appréhension de la forme dans l'âme, et un modèle davantage tourné vers le corps, comme celui de Galien. C'est pourquoi, d'ailleurs, la cure joue sur les deux tableaux : d'un côté, il convient de reprendre une activité sexuelle, de boire du vin, de prendre des bains ; de l'autre, il faut entretenir des conversations amicales, écouter de la musique ou la récitation de poèmes (versus recitatio). La fin du texte insiste toutefois sur le remède le plus efficace : s'entourer d'amis dont la beauté, le savoir et la morale permettent d'échapper aux effets dévastateurs de cette forme de mélancolie ${ }^{18}$. Il ne s'agit donc pas seulement de substituer une

15 Le texte est édité dans Wack, Lovesickness in the Middle Ages, 188-193.

16 Pour une vue d'ensemble des sources, cf. J. L. Lowes, « The Loveres Maladye of Hereos », Modern philology 11 (1914), n4, 491-546; M. McVaugh, «Introduction », Arnaud de Villeneuve, De amore heroico, dans Arnaldi de Villanova Opera medica omnia, vol. III, Barcelone 1985, 11-39 (en partic. 14-21). 17 Constantin l'Africain, Viaticum, I, 20, dans Wack, Lovesickness in the Middle Ages, 186 : «Est autem magnum desiderium cum nimia concupiscentia et affectione cogitationum. Unde quidam philosophi dicunt : Eros est nomen maxime delectationis designativum. Sicut autem fidelitas est dilectionis ultimitas, ita et eros delectationis quedam est extremitas. »

18 Ibid., 192 : «Quod perfectissimum sibi esse dinoscitur si boni consocii aggregentur qui et in pulchritudine valeant scientia vel moribus. Dictum est enim quia maxima est delectatio ut vinum bibatur et colloqui sapientibus. » 
belle forme dans l'âme à une autre, mais de recouvrer le gouvernement de son corps par une réforme intellectuelle et morale, réforme qui ne peut s'accomplir dans la solitude à laquelle confine la mélancolie. La guérison requiert un banquet.

La traduction du Canon d'Avicenne par Gérard de Crémone à la fin du XII ${ }^{\mathrm{e}}$ siècle permit aux Latins de trouver des compléments utiles pour rendre compatibles ces deux manières d'aborder l'amour, celle de Platon et celle de Galien. Pour cette raison, il fut rapidement cité par certains commentateurs du Viaticum, par Arnaud de Villeneuve dans le traité De amore heroico ou encore par Dino del Garbo dans son commentaire à Donna me prega ${ }^{19}$. C'est dans la première fen du troisième livre du Canon, entièrement consacrée aux " maladies de la tête », que le quatrième traité comporte un chapitre intitulé de hylisci, translittération de l'arabe al'ishq, signifiant précisément ce mal qui suit une pensée immodérée de l'être aimé ${ }^{20}$. Ce cas très particulier est traité parmi un ensemble de maladies qui atteignent « les opérations sensibles et les mœurs » : la léthargie (subeth), l'aliénation mentale (alienatio), la folie (mania), et diverses formes de mélancolie, y compris l'étrange et inquiétante lycanthropie (cucubuth).

Considérant tour à tour chacune de ses maladies, Avicenne détaille les causes physiques qui peuvent intervenir au niveau des sens externes (vue, odorat, ouïe, toucher, goût) ou internes (mémoire, imagination, faculté estimative ou cogitative de l'âme). Pour ce qui est de la mélancolie en général, Avicenne insiste cependant fortement sur la dimension mentale de cette maladie. Il s'agit d'abord « d'un changement dans les jugements et les pensées par rapport à leur cours naturel », qui entraîne un changement dans la complexion et les humeurs du cerveau ${ }^{21}$. Autrement dit, c'est parfois la pensée elle-même, avec ses brusques changements, qui entraîne une modification de l'équilibre du corps, et du cerveau en particulier, siège des fonctions cognitives de l'âme, à l'exception de l'intellect. Il y aurait donc comme un cercle vicieux, partant de la pensée, pour passer par le corps et affecter à nouveau la pensée elle-même. Quant au mal d'amour en particulier, il s'agit « d'un tourment mélancolique, [ou] semblable à la mélancolie, dans lequel l'homme est amené de lui-même, dans l'instant, à une excitation de la pensée dirigée vers la beauté de certaines formes et figures qui sont en lui $»^{22}$. L'amour,

19 Par exemple : Dino del Garbo, Scriptum super cantilena Guidonis Cavalcantibus, dans Fenzi, La Canzone d'amore di Guido Cavalcanti, 116.

20 Sur le sens de ce terme, cf. Wack, Lovesickness in the Middle Ages, 35-38.

21 Par commodité nous citons le texte d'Avicenne dans l'édition du commentaire de Gentile da Foligno faite à Venise en 1520. Ici f. 84rb.

22 Avicenne, Canon, III, 1, 4, 22, f. 90rb : « Hec egritudo est solicitudo melancholica similis melancholie in qua homo sibi iam induxit incitationem cogitationis sue super pulchritudine quarundam formarum et figurarum que insunt ei. » 
comme la mélancolie en général, est donc d'abord un tourment de la pensée ellemême ou, comme le précise Avicenne, du jugement de la faculté estimative. Grâce à cette fonction, en effet, nous jugeons les objets de connaissance sous l'angle de l'agréable ou du désagréable, du bon ou du mauvais ${ }^{23}$. C'est de ce jugement, partagé par d'autres espèces animales, que naît le désir. Par conséquent, lorsqu'un élément de ces rouages se trouve modifié, qu'il s'agisse du sens, de l'imagination, ou de l'estimation, c'est toute la chaîne qui est affectée, jusqu'au corps lui-même, qui subit certains changements physiques visibles. C'est donc d'abord, comme chez Platon, la pensée d'une belle forme qui engendre un changement dans le corps. En cela, l'amour maladif ne diffère pas, dans sa définition minimale, d'autres formes plus positives d'amour. Il devient un mal lorsqu'une erreur intervient dans le processus d'appréhension de la forme. Comme chez Galien cette fois, c'est le cerveau, siège des puissances de l'âme, qui va rendre la pensée obsessionnelle et maladive une fois sa complexion affectée, notamment par l'intensité du désir engendré par la contemplation de la forme.

Le texte d'Avicenne est dense et concis. Après avoir défini la passion amoureuse, il en précise les symptômes : profondeur et sécheresse des yeux, absence de larmes, soupirs, ou encore réactions extrêmes à l'écoute de chansons d'amour (amoris cantilenas). Sur ce dernier point, Avicenne précise que certains malades rient aux éclats en entendant de telles chansons, quand d'autres se mettent à pleurer, en particulier s'ils se remémorent la distance qui les sépare de l'être aimé $^{24}$. Vient ensuite la cure, essentiellement fondée sur la distraction du malade, obtenue par un travail sur l'imagination. Il faut que sa pensée se porte vers d'autres objets que l'être aimé. Les conseils se font alors plus pratiques. Comme chez ses prédécesseurs, un premier remède consiste à avoir des relations sexuelles, avec qui que ce soit d'ailleurs, dans les limites autorisées par la foi et la loi, précise Avicenne. Il est aussi possible de se servir de l'art des vetulae, les petites vieilles, ces sorcières qui savent raconter des horreurs aux malades, et les détournent ainsi de leur monomanie. Enfin, comme pour y insister à nouveau, le texte revient sur le fait que certaines personnes peuvent trouver de l'aide dans l'écoute de chansons (cantilenas) ${ }^{25}$. Il n'est donc plus fait mention de l'aspect moral de la cure. Certes, la parole occupe encore une place de premier choix, mais c'est en tant qu'elle peut agir sur l'imagination qu'elle est ici mise en avant,

23 On trouvera une brève présentation du système des sens internes chez Avicenne dans C. Di Martino, Ratio particularis : doctrine des sens internes d'Avicenne à Thomas d'Aquin, Paris 2008. 24 Avicenne, Canon, III, 1, 4, 22, Venise, 1520, t. 3, f. 90va.

25 Ibid., f. 91ra : «Et quidam homines sunt quos sanat letitia et auditus cantilene. Et quidam sunt quos illud augmentat in hylisci. » 
non parce qu'elle remettrait le malade sur le droit chemin. C'est à la poésie et non à la rhétorique morale qu'il faut recourir pour guérir ceux qui souffrent de ce mal.

Cet appel récurrent à la poésie n'est pas nouveau, puisque le Viaticum de Constantin l'Africain mentionnait déjà la récitation de poèmes parmi les remèdes. D’ailleurs, le terme arabe ( $a$ ' ish $q$ ) choisi pour désigner ce mal est emprunté à la littérature amoureuse ${ }^{26}$. Mais la précision d'Avicenne est intéressante : le pouvoir guérisseur de la poésie n'est pas automatique, puisque les poèmes peuvent parfois aggraver l'état de l'amant. Cette différence s'explique par les mécanismes précis qui régissent l'action de l'âme sur le corps. Gentile da Foligno a fort bien compris cela dans son commentaire, ce qui n'est pas négligeable, puisque c'est le seul que nous possédions pour le Trecento.

\section{Le commentaire de Gentile da Foligno : un hapax}

Les plus anciens statuts de l'Université de Bologne concernant l'enseignement de la médecine, datés de 1405, précisent que le livre III du Canon était inscrit au programme, à raison de trois ou quatre fen chaque année pendant quatre ans ${ }^{27}$. Nous ne connaissons pourtant que sept commentaires au livre III entre le XIV et le $\mathrm{XV}^{\mathrm{e}}$ siècle :

- Mondino de’ Liuzzi (m. 1326)

- Niccolò Bertruccio (fin XIIIe s.-1347)

- Magister Peronus ( $\left(^{\text {er }}\right.$ XIV $\mathrm{S}$.)

- Gentile da Foligno (v. 1280-1348)

- Tommaso del Garbo (m. 1370)

- Ugolino da Montecatini (v. 1345-1425)

- Jacques Despars (v. 1380-1458)

Parmi eux, seuls trois auteurs ont commenté les premières fen du livre III : Niccolò Bertruccio et Gentile da Foligno en Italie au XIV ${ }^{\mathrm{e}}$ siècle, Jacques Despars en France au XVe siècle ${ }^{28}$. Du commentaire de Niccolò Bertruccio nous n’avons conservé que quelques fragments ${ }^{29}$, ainsi que le résumé qu'il en donne dans son Collectorium totius medicine. Malheureusement, ni les fragments, ni le Collectorium n'abordent

26 Voir les remarques de Wack, Lovesickness in the Middle Ages, 35-38.

27 C. Malagola, Statuti delle Università dei Collegi dello Studio bolognese, Bologne 1888, 275-277.

28 Il est possible que Mondino de' Liuzzi ait aussi commenté cette partie, mais nous ne possédons aucun témoin de son commentaire.

29 Ms. Cesena, Biblioteca Malatestiana, S.XXVII.4, ff. 63ra-102rb. 
la maladie d'amour. Aussi le commentaire de Gentile da Foligno au chapitre sur le mal d'amour est-il le seul connu, en Italie, au XIV siècle.

Le commentaire de Gentile da Foligno au chapitre 22 de la fen 1, Traité IV du livre III du Canon est conservé dans six manuscrits ${ }^{30}$, ainsi que dans les éditions de 1477 à Padoue et de 1522 à Venise.

- Città del Vaticano, Biblioteca Apostolica Vaticana, Vat. Lat. 4459, ff. 72rb-vb + f. volant

- Città del Vaticano, Biblioteca Apostolica Vaticana, Vat. Lat. 2475, ff. 131va-132ra (XV' siècle)

- Città del Vaticano, Biblioteca Apostolica Vaticana, Vat. Lat. 2483, f. 168va-b (daté de 1388 pour le texte de Gentile)

- Città del Vaticano, Biblioteca Apostolica Vaticana, Pal. Lat. 1130, ff. 82ra84ra (première moitié du XVe siècle)

- München, Bayerische Staatsbibliothek, Clm 244, ff. 78ra-va (XIV siècle)

- Mantova, Biblioteca comunale B.V.14 (262) (nous n'avons pas pu consulter ce témoin à ce jour ; la fen 1 occupe les ff. 1ra-167vb)

Comme l'a montré Joël Chandelier, le manuscrit Vaticanus Latinus 4459 est en grande partie autographe ${ }^{31}$. Ce témoin est d'autant plus important et intéressant qu'il inclut des ajouts tardifs de l'auteur, parfois insérés dans des feuillets volants, dont certains sont datés des années 1342-1347. Or il se trouve que Gentile da Foligno a justement ajouté une partie à la première version (entre 1332 et 1338) de son commentaire au chapitre sur l'amour. À la suite du commentaire lemmatique, on trouve en effet une longue question disputée sur le pouvoir des mots, des incantations et des amulettes portées autour du cou, dont une partie date de la première rédaction, l'autre d'une seconde mouture.

Dans la version antérieure à 1338, on trouve simplement une brève dubitatio : « étant donné qu'Avicenne a dit dans ce chapitre que les paroles sont efficaces, un doute s'abat sur nous : les paroles, les incantations, les amulettes portées au cou et les caractères ont-ils un pouvoir ${ }^{32}$. À ce stade de la rédaction, Gentile

30 Il existe des témoins contenant d'autres parties du livre III, sans ce chapitre.

31 Chandelier, Avicenne et la médecine en Italie, I, 3.

32 Gentile da Foligno, Comm. Canon, III, 1, 4, 22, Ms. Vat. Lat. 4459, f. 72va : « Sed quia Avicenna dixit quod verba in hoc capitulo conferunt, ideo cadit dubitatio an verba et precantationes et colli suspensiones valeant sive brevia. » Le terme brevia est difficile à traduire. Nous avons choisi « caractères ", au sens de caractères magiques, faute de mieux, en suivant certains usages répértoriés dans C. Du Cange et al., Glossarium mediae et infimae latinitatis, Niort 1883-1887, t. 1, col. 745a. De plus, on peut lire dans le ms. Pal. Lat. 1225, f. 456ra : « Utrum incantationes, karakteres et husiusmodi talia valeant. » 
da Foligno se contente d'une courte introduction dialectique, formée d'une série d'arguments pro et contra, sans determinatio. La solution, accompagnée d'un long développement, se trouve dans l'ajout ultérieur, qui s'étend dans le manuscrit autographe sur la seconde colonne du verso du folio 72, puis sur un feuillet volant $(73 r-v)$.

Cette intervention dans le manuscrit original eut des conséquences sur la transmission du texte, qui a circulé sous deux formes distinctes, celle de 1338 et celle qui fut amendée plus tard. Il est intéressant de remarquer que les éditions de la Renaissance, généralement utilisées par les historiens, ne contiennent que la première version inachevée. Par ailleurs, outre le Vat. Lat. 4459, seul le manuscrit Pal. Lat. 1130, datant du XV siècle, contient la quaestio dans son intégralité. Le reste de la tradition reproduit l'état du texte avant 1338. Il est vrai que l'apparition de cette dubitatio à cet endroit du commentaire, même dans sa version inachevée, avait de quoi surprendre. Ainsi, le scribe du manuscrit Vat. Lat. 2475 (f. 132ra) se contente de recopier l'intitulé de la question et d'ajouter qu'elle n'a rien à faire à cet endroit du commentaire. Dans le manuscrit de Munich, CLM 244, on peut lire à la fin de la partie dialectique contenue dans la version de 1338 : « Le Conciliator [Pietro d'Abano] récite les nombreux modes [d'action de la parole], va donc le voir, ainsi que la question que nous avons produite sur le sujet ${ }^{33}$, ce qui indique que le scribe avait sans doute connaissance d'une discussion plus longue sous forme de quaestio. Ce témoin semble, en outre, mettre en doute l'originalité de Gentile da Foligno par rapport à Pietro d’Abano, lequel a en effet examiné la même quaestio dans la differentia 156 de son Conciliator.

L'existence d'une dispute indépendante sur le pouvoir des incantations semble corroborée par une autre tradition, manuscrite et imprimée. Trois manuscrits, ainsi qu'une édition renaissante, conservent en effet cette quaestio indépendamment du commentaire au Canon.

- Torino, Biblioteca nazionale, *F. II. 2, ff. 87va-88ra (1467-1471).

- Città del Vaticano, Biblioteca Apostolica Vaticana, Pal. Lat. 1225, f. 456ra-vb (XV's.).

- Wolfenbüttel, Herzog August Bibliothek 2794, ff. 290r-91r (XV s., 1432-1433).

\section{Editions :}

- Quaestiones et tractatus extravagantes, Venise, 1526, q. 55, f. 108ra-vb.

33 Gentile da Foligno, Comm. Canon, III, 1, 4, 22, Ms. Münich, Bayerische Staatsbibliothek, Clm 244, f. 78va : « Recitat Conciliator [in marg. Differentia 156] modos multos et igitur vide eum et questionem meam quam fecimus (pro feci?) de illo]. » Les éditions de la Renaissance font de même et renvoient elles aussi à la differentia 156 du Conciliator de Pietro d'Abano qui traite en effet du même sujet. 
Les trois manuscrits attribuent sans équivoque le texte à Gentile da Foligno et leur contenu correspond exactement au texte que l'on trouve dans le manuscrit autographe de la bibliothèque vaticane.

$\mathrm{Au}$ vu de la situation des manuscrits, il est difficile de savoir si Gentile da Foligno a seulement disputé cette quaestio à l'occasion de son enseignement sur le Canon, une première fois partiellement avant 1338, puis plus précisément quelques années plus tard, ou s'il l'a disputé deux fois, dans deux contextes différents, en commentant le Canon et lors d'une disputatio indépendante. Dans le premier cas, la version remaniée dans le Canon a pu circuler de manière indépendante. Dans le second cas, Gentile da Foligno aurait simplement inséré le texte d'une de ses questions disputées dans son exemplaire du commentaire au Canon. D'un côté nous avons l'évocation d'une quaestio dans un des manuscrits du commentaire au livre III, de l'autre, l'incipit du manuscrit de Turin indique une dubitatio et non une quaestio, comme s'il copiait simplement la version complète du commentaire ${ }^{34}$ et le ms. Pal. Lat. 1225 ajoute dans les arguments quod sic une citation du chapitre du Canon sur l'amour, comme pour en préciser l'origine $^{35}$.

Quoi qu'il en soit, le commentaire de Gentile da Foligno est doublement exceptionnel : d'abord, parce qu'il est unique dans le paysage intellectuel italien du XIV siècle ; ensuite, parce qu'il traite la question de manière originale en $\mathrm{y}$ annexant cette discussion sur le pouvoir des mots et des démons. Commençons par le commentaire littéral du texte d’Avicenne.

\section{La passion amoureuse entre fureur divine et mélancolie}

Le commentaire de Gentile da Foligno, bien qu'il se veuille proche de la lettre d'Avicenne, s'en écarte de manière importante sur plusieurs points. Premier aspect digne d'intérêt, le médecin de Pérouse propose une explication étymologique rare de l'expression amor hereos, utilisée depuis longtemps par les médecins pour désigner la passion amoureuse, et traduisant ici l'arabe al'ishq. Dans la traduction du Viaticum d'Ibn al-Jazzār, on peut lire que « l'amour, qui est appelé

34 Gentile da Foligno, Quaestio, Ms. Torino, Biblioteca Nazionale, F. II. 2, f. 87va : « Est dubitatio an verba et precantationes et colli suspensiones valeant... ».

35 Ms. Pal. Lat. 1225, f. 456ra : «Et videtur quod sic, primo per Avicennam $3^{\circ}$ Canonis fen prima capitulo de ilisci, cap. 22, quod idem est, quod amor sive ereos, ubi dicit quod verba in hoc capitulo conferunt. » 
eros, est une maladie qui touche le cerveau $»^{36}$. Par une série de transformations sémantiques, les manuscrits ont rapidement changé eros en heros, parfois herus, puis en hereos, voire heroicus ${ }^{37}$, rapprochant ainsi l'amour grec de l'amour masculin et noble des héros de la littérature courtoise, tel qu'il est codifié par le $D e$ amore d'André le Chapelain ${ }^{38}$. La passion amoureuse toucherait donc d'abord le cerveau des hommes d'un certain rang social. Une telle interprétation, qui rappelle lointainement un passage du Cratyle (398c-e) de Platon ${ }^{39}$, se lit dès le premier commentaire latin du Viaticum composé à Paris par Gérard de Berry, un contemporain d'André le Chapelain. Glosant le terme heros, il écrit : « on appelle héros les hommes nobles, qui par leurs richesses et leur vie voluptueuse souffrent plus de cette passion ${ }^{40}$. On la retrouve ensuite dans quelques commentaires au Viaticum $^{41}$, mais aussi dans le De amore heroico d'Arnaud de Villeneuve ${ }^{42}$ ou dans le commentaire de Jacques Despars au Canon d'Avicenne, au XV $\mathrm{Xiècle}^{43}$.

Gentile da Foligno introduit quant à lui une rupture franche dans cette tradition, en écartant ce lien entre amour et noblesse, pour lui préférer le thème platonicien de la fureur divine :

En premier lieu, il convient de considérer que cette passion est appelée illisci par les arabes et que nous l'appelons amour, ou passion qui assaille l'homme en raison de l'amour. Et

36 Constantin l'Africain, Viaticum, I, 20, dans Wack, Lovesickness in the Middle Ages, 186.

37 Sur l'histoire de ces transformations, voir Lowes, " Maladye of Hereos » et D. Jacquart et C. Thomasset, «L'amour 'héroïque' à travers le traité d'Arnaud de Villeneuve », dans La folie et le corps, Etudes réunies par Jean Céard, Paris 1985, 143-158.

38 Pour plus de détails sur les rapports entre analyse médicale et littérature courtoise, cf. M.F. Wack, « The Liber de heros morbo of Johannes Afflacius and its Implications for Medieval Love Conventions », Speculum 62 (1987), 324-344.

39 Comme le rappelle Giorgio Agamben (Agamben, Stanze, 195), dans le Cratyle Platon faisait dériver héros et eros. Il mentionne aussi le commentaire de Hiéraclès aux Vers dorés attribués à Pythagore où l'on trouve aussi cette association.

40 Gérard de Bérry, Glosulae super Viaticum, dans Wack, Lovesickness in the Middle Ages, 202 : «Amor qui eros. Heroes dicuntur viri nobiles qui propter divicias et mollitiem vite tali potius laborant passione. »

41 Par exemple le commentaire (version B) de Pierre d'Espagne, ibid., 232 : « Et ita fit hec passio que amor hereos vocatur ab heremis, id est nobilioribus quia maxime solent incurrere istam passionem.»

42 Arnaud de Villeneuve, De amore heroico, dans McVaugh, Arnaldi de Villanova, 50-51 : «Dicitur autem amor heroicus quasi dominalis, non quia solum accidit dominis, sed aut quia dominatur subiciendo animam et cordi hominis imperando, aut quia talium amantium actus erga rem desideratam similes sunt actibus subditorum erga proprios dominos. »

43 Jacques Despars, comm. Canon, III, 1, 4, 23, Lyon, 1498, f. Niiirb : « Et dicitur hereos vel hereosus is insanus amor, quia plus accidit viris nobilibus et viris heroicis quam viris simplicibus de communi plebe. » 
on l'appelle hereos, c'est-à-dire passion hérotique (passio herotica), c'est-à-dire divine, soit parce que certains ont estimé que cette passion était produite par Dieu, comme cela apparaît dans le septième commentaire [de Galien] au premier livre des Pronostics [d'Hippocrate], soit parce que ceux-là [qui en souffrent] sont détachés de toute autre affaire humaine, comme cela arrive chez ceux qui contemplent les choses divines ${ }^{44}$.

L'expression passio herotica, présente dans le manuscrit autographe de Gentile da Foligno, est rare. Autant que nous le sachions, il faut attendre le XVI siècle pour retrouver un usage d'un terme proche (heroticos) dans un texte de Daniel Sennert consacré à l'amor hereos ${ }^{45}$. Du reste, si l'expression a bien été reprise dans les éditions de la Renaissance ${ }^{46}$, certains témoins manuscrits l'ont remplacée par heroica, rattachant ainsi notre auteur à la tradition ${ }^{47}$. Mais si Gentile avait voulu écrire heroica et non herotica, comment interpréter l'intrigante précision : passio herotica, id est divina ? Plusieurs pistes de lecture peuvent être proposées.

Tout d'abord, le néologisme de Gentile da Foligno n'est pas sans rappeler la fureur divine des dialogues platoniciens. Une des sources possibles serait la traduction latine du traité de Soranus intitulé Maladies aiguës, maladies chroniques par Caelius Aurélien, dans laquelle le médecin romain utilise un terme tout à fait proche de celui choisi par Gentile, précisément lorsqu'il est question de maladie d'amour ${ }^{48}$. Là, Caelius Aurélien citait les stoïciens, mais aussi et surtout le Phèdre (265b) de Platon, où il est dit que la fureur (furor) est double : l'une est d'origine corporelle, l'autre est divine. Suivant de près le texte platonicien, le médecin résumait la théorie des quatre sous-espèces de fureur divine : l'une viendrait d'Apollon, l'autre de Dionysos, l'autre des Muses, une autre enfin, l'amour, vient d'Aphrodite et d'Eros. Cette dernière est appelée eroticon dans le texte de Caelius Aurélien. Or, pour Platon, cette folie amoureuse venue d'Eros est bonne, c'est même la meilleure, et l'on ne doit pas la confondre avec le « contre amour » (anteros), véritable maladie du corps (Phèdre, 255d-e). Cette maladie divine était donc entourée d'une connotation éminemment positive dans la philosophie platonicienne.

44 Gentile da Foligno, comm. Canon, III, 1, 4, 22, Vat. Lat. 4459, f. 72rb : « Considerandum primo quod hec passio vocatur hylissci apud arabes et vocatur apud nos amor, sive passio, quam homo propter amorem incurrit. Et vocatur hereos, id est passio herotica, id est divina, quia quidam putaverunt a deo fieri hanc passionem, ut primo Pronosticorum commento 7, vel quia isti sunt abstracti ab omnibus aliis negociationibus, sicut accidit in contemplatoribus divinorum. »

45 Cf. Lowes, "Maladye of Hereos ", 45. Au XVI siècle toujours, Pierre Forestus parle lui de " passion divine », comme notre médecin.

46 L'édition de 1477 (f. 114ra) l'a transformé en errotica, mais celle de 1520 a conservé herotica.

47 Par exemple : Ms. Münich, Bayerische Staatsbibliothek, Clm 244, f. 78ra.

48 Caelius Aurelianus, Maladies aiguës, maladies chroniques, I, 5, éd. et trad. anglaise I.E. Drabkin, Chicago 1950, 144. Certains témoins indiquent même heroticon, avec un « h », comme c'est encore le cas dans l'édition contenue dans les Medici antiqui omnes, Venise 1547, f. 257. 
Recommandé par Cassiodore et très lu durant le haut Moyen Âge, le texte de Caelius Aurélien est rarement cité aux XIII ${ }^{\mathrm{e}}$ et $\mathrm{XIV}^{\mathrm{e}}$ siècles. Il serait donc quelque peu surprenant que Gentile da Foligno soit allé chercher la thèse platonicienne dans ce texte tombé en désuétude. Certes, le thème de la fureur divine se trouve chez nombre d'auteurs de l'Antiquité tardive, et certains, comme Aulu-Gelle (Nuits attiques, XVIII, 7), rapprochaient la mélancolie et les vertus héroïques, ce qui aurait pu permettre, assez tôt dans l'histoire, un croisement de la tradition platonicienne avec celle du célèbre Problème XXX, 1 du Pseudo-Aristote ${ }^{49}$. Mais poser en ces termes l'équation entre Eros et maladie divine était plus que rare dans la médecine médiévale.

On trouve cependant une autre étymologie intéressante au XIII ${ }^{\mathrm{e}}$ siècle, rare elle aussi, dans le Fabularius de Conrad de Mure. Alors qu'il énumère diverses formes d'odes, il désigne l'une d'elles par le mot herotice. Ce nom, qui dériverait de la muse Erato (ou Herato), muse de la poésie lyrique et érotique, signifierait simplement « avec amour » ou passionnément (amatorie) $)^{50}$. Une telle association repose sur un passage des Remèdes d'amour d'Ovide ${ }^{51}$, l'un des textes les plus fréquemment cités dans les discussions médiévales sur le mal d'amour, y compris par les médecins ${ }^{52}$. La passion amoureuse pourrait ainsi être rapprochée de la fureur poétique du Phèdre de Platon, celle qui inspire les poètes, notamment dans le domaine amoureux, thème qui sera repris sous une autre forme dans le Problème XXX,1 du Pseudo-Aristote. Aussi, la passion « hérotique » pouvait-elle évoquer ensemble l'amour, l'héroïsme et l'inspiration poétique.

Ce ne sont là que conjectures difficiles à étayer. La fin de la citation de Gentile da Foligno donne tout de même un nouvel éclairage sur le sens de cette étymologie

49 On trouve aussi l'adjectif erotikoi dans le Problème XXX, 1 du Pseudo-Aristote, mais la traduction latine de Barthélémy de Messine a traduit ce terme, de manière assez étrange, par interrogativi. Voir sur ce point les remarques de P. De Leemans et M. Goyens, « La transmission des savoirs en passant par trois langues : le cas des Problemata d'Aristote traduits en latin et en moyen français », dans P. Nobel (éd.), La transmission des savoirs au Moyen Âge et à la Renaissance. [Actes du colloque international organisé à Besançon et à Tours, du 24 au 29mars 2003], T. 1 : Du XII au $X V^{e}$ siècle, Besançon 2005, 231-257 (en partic. 239-240).

50 Conrad de Mure, Fabularius, éd. T. van de Loo, Turnhout 2006, 324 : « Et nota, quod odarum quedam inscribitur 'pragmatice', quedam 'proseutice', quedam 'paranetice', quedam 'prosphonetice' seu 'prophonetice', quedam 'ypothetice', id est personaliter uel suppositiue, quedam 'herotice', id est amatorie, uel 'herotice' ab Herato, que est una de nouem Musis et interpretatur simile - Ouidius de arte : 'Ac Herato, nam tu nomen amoris habes.' »

51 Ovide, Ars amatoria, 2,15-16 : « Nunc mihi, si quando, puer et Cytherea, fauete : / nunc Erato, nam tu nomen Amoris habes. »

52 Voir sur ce point D. Jacquart, « La maladie et le remède d'amour dans quelques écrits médicaux du Moyen Âge », dans D. Buschinger et A. Crépin (éds.), Amour, mariage et transgression au Moyen Âge, Göppingen 1984, 93-101. 
d'inspiration platonicienne, puisque deux interprétations y sont proposées : soit cette passion est d'origine divine (Dieu en est la cause), soit elle rapproche du divin, en amenant l'individu à se détacher des choses mondaines (Dieu est la fin).

La première option, proche de celle énoncée par Platon dans certains discours du Banquet et dans le Phèdre, ne semble guère prise au sérieux par notre auteur, qui se contente de renvoyer, quoique de manière paradoxale, à Galien. Un tel renvoi serait contre-productif s'il devait fonder cette définition ou la faire accepter, puisque dans son commentaire aux Pronostics d'Hippocrate, le médecin de Pergame s'efforçait précisément de limiter l'explication médicale à des causes strictement naturelles, suivant en cela l'exemple de son illustre prédécesseur ${ }^{53}$. On se souvient, en effet, que le traité hippocratique De la maladie sacrée dénonçait justement, à propos de l'épilepsie, l'idée d'une origine divine des maladies, réduisant cette théorie à celle des charlatans et des magiciens ${ }^{54}$. Il est vrai cependant que le texte des Pronostics commenté par Galien et mentionné par Gentile da Foligno est ambigu, puisqu'il demande au médecin de ne pas négliger l'existence d'un élément « divin » dans la maladie lors de son pronostic ${ }^{55}$. Ce passage a fait l'objet de nombreux commentaires, de l'Antiquité jusqu'à nos jours, puisqu'il semble en contradiction avec ce que dit Hippocrate ailleurs ${ }^{56}$. Galien, en tout cas, lorsqu'il commente ce passage, mentionne l'épilepsie et sa prétendue origine divine, mais aussi la maladie d'amour. Après avoir rappelé que l'on ne trouve nulle part l'idée d'une origine divine des maladies dans les textes du médecin de Cos, sa propre solution consiste à dire que par « divin » ou « céleste » il faut plutôt entendre la qualité de l'air, dans le ciel, ce qui serait cohérent avec le traité Airs, eaux, lieux. Quant au mal d'amour, Galien ajoute que l'interprétation platonisante se serait propagée à partir de l'histoire d'amour improbable entre Antiochus et sa belle-mère Stratonice, racontée par tant d'auteurs antiques - notamment ValèreMaxime et Plutarque. Ces récits mettent en scène le médecin Erasistrate découvrant cet amour grâce à la variation du pouls chez le jeune prince $\mathrm{e}^{57}$. À partir de

53 Cf. J. Jouanna, « Hippocrate de Cos et le sacré », Journal des savants 1-2 (1989), Janvier-Juin, $3-22$.

54 Sur la «maladie sacrée », voir O. Temkin, The Falling Sisckness : A History of Epilepsy from the Greeks to the Beginnings of Modern Neurology, Baltimore 1994 (en partic. 3-27).

55 Hippocrate, Pronostic, éd. et trad. J. Jouanna, Paris 2013, 3.

56 Voir les remarques de J. Jouanna dans Hippocrate, Pronostics, LVI-LXI.

57 Galien, In librum Hippocratis Pronosticorum, in Thaddei Florentini expositiones in divinum pronosticorum Ipocratis librum, Venise 1527, f. 197ra-b : « Cecidit inter expositores huius libri, intentione hac, quam nominavit Hippocrates celestem [...] quia quibusdam eorum videtur quod egritudines accidunt hominibus ex ira dei [...]. Nos non invenimus Hippocratem dicere in librorum aliquorum suorum quod deus benedictus et sublimis causat aliquam egritudinem. [...] Non ergo oportet ut dicatur in egritudine epilepsie quod ipsa est egritudo celestis neque in amore. 
là, on aurait glosé sur l'origine divine de cet amour. Pour d'autres, ajoute Galien, cette interprétation proviendrait simplement de croyances et de proverbes populaires présentant l'amour comme une manifestation de Satan ${ }^{58}$. Les lecteurs médiévaux de ce texte ne s'y trompaient pas et reprenaient généralement à leur compte l'explication de Galien ${ }^{59}$.

La seconde option, qui ne se trouve ni chez Hippocrate ni chez Galien, peut elle-même faire l'objet d'au moins deux interprétations. Une première lecture consisterait à y voir une analogie de caractère entre l'amoureux, le mélancolique et le religieux, analogie qui pourrait trouver son origine dans le traité Sur la mélancolie d'Ishāq ibn Imrān (vers 900), traduit en latin par Constantin l'Africain $\mathrm{au} \mathrm{XI}{ }^{\mathrm{e}}$ siècle. Il y est écrit que :

Très nombreux sont les hommes saints et pieux qui deviennent mélancoliques à cause de leur grande piété et de leur crainte de la colère divine, ou à cause de leur désir ardent de Dieu, qui finit par dominer et vaincre leur âme ; toute leur âme et leurs pensées sont préoccupées uniquement de Dieu, de la contemplation de Dieu, sa grandeur et l'exemple de sa perfection. Ils tombent dans la mélancolie comme des amoureux ou des voluptueux, ce qui nuit autant aux capacités de l'âme qu'à celles du corps, car de l'une dépend l'autre. ${ }^{60}$

Dans ce texte, en partie inspiré par les thèses de Rufus d'Éphèse et par le célèbre Problème XXX, 1 du Pseudo-Aristote, la mélancolie des hommes pieux devient proche de celle des amoureux du fait qu'ils subissent tous deux les effets d'une pensée obsessionnelle. Tous deux se détachent de la vie mondaine, comme les mélancoliques. Ou bien, devrait-on plutôt dire : parce qu'ils se détachent des occupations humaines, ils sont pris d'un accès de mélancolie. On retrouve donc

Quidam enim homines estimaverunt quod egritudo celestis est amor, et testificantur super illud ex enunciatione certa significationis. Eristratis super egritudinem filii regis, quod causa eius fuit amor. »

58 Plus loin, Galien dit en effet qu'il n'y a pas de raison d'attribuer cette maladie à une divinité « nisi suscipiamus sermonem antiquorum proverbiorum : ipsi enim estimant quod amor accidit quibusdam hominibus propter Sathan. »

59 C'est le cas, par exemple, de Taddeo Alderotti, qui refuse explicitement de faire de l'amour une maladie divine. Cf. T. Alderotti, Expositio in divinum pronosticorum Ipocratis librum, in Thaddei Florentini expositiones, f. 197va : « Duplex cadit expositio super hoc verbo : prima quarum est, quia credunt quidam quod deus faciat venire egritudines aliquibus ; unde vocant signum celeste considerationem in divinis ; unde ipsi ponunt quod egritudo amoris et epilepsia sunt egritudines divine, eo quod deus mittat eas. Et ista expositio mala est [...] cum ipse nunquam dixerit quod deus mittat alicui aliquam egritudinem [...]. Secunda vero est quod per signum celeste possumus intelligere dispositionem aeris [...]. »

60 Ishāq ibn Imrān, De melancolia, trad. Latine de Constantin l'Africain, dans Opera, vol. I, 283, cité et traduit dans R. Klibansky, E. Panofsky et F. Saxl, Saturne et la mélancolie. Etudes historiques et philosophiques : nature, religion, médecine et art, Paris 1989, 146. 
un des thèmes du Problème XXX, 1 : la mélancolie devient un trait du génie, du sage et du poète, personnages qui sont, d'une certaine manière, hors du monde ; mais la mélancolie est aussi à l'origine d'une passion érotique, voire d'une forme de luxure ${ }^{61}$. On trouve cette même idée, selon laquelle les religieux subissent particulièrement le mal d'amour, chez Guillaume de Brescia, un élève de Taddeo Alderotti et médecin personnel de Clément V puis de Jean XXII. Dans sa Practica, il n'hésite pas à affirmer que ce mal touche ceux qui vivent dans le plaisir, les solitaires, les oisifs, mais aussi les religieux et les nonnes ${ }^{62}$. Le détachement de l'amoureux serait donc une forme d'enfermement dans la pensée, dans l'imagination, pareil à celui des religieux qui contemplent le divin.

Une seconde interprétation, plus positive, consisterait à voir dans ce bref passage une allusion à une forme supérieure d'amour visant Dieu lui-même. Divin, le mal d'amour le serait en ce sens qu'il permet de s'élever vers Dieu en quittant les affaires mondaines. Le vocabulaire de la passion divine se lit d'ailleurs chez un Thomas d'Aquin, par exemple, lorsque celui-ci évoque l'amour de Dieu, au sens du génitif objectif ${ }^{63}$. Dans ce cas, Gentile da Foligno - ou l'opinion qu'il cite - s'opposerait frontalement à une longue tradition, qui prend sa source dans les textes patristiques et se poursuit dans la théologie médiévale, selon laquelle le mélancolique, comme celui qui souffre d'acedia, serait incapable d'accéder à la métaphysique et, par suite, à une pensée de Dieu ${ }^{64}$.

Une dernière voie mérite toutefois d'être explorée. Elle recoupe d'ailleurs, au moins partiellement, les précédentes. Comme l'on sait, la question de l'origine du mal d'amour (maladie humaine ou maladie divine ; origine divine ou mouvement vers Dieu) fut l'un des thèmes de prédilection de la poésie italienne des XIII ${ }^{\mathrm{e}}$ et $\mathrm{XIV}^{\mathrm{e}}$ siècles. Gentile da Foligno, familier de cette tradition, ne souhaitait-il pas intervenir, au moins indirectement, dans ce débat ? Plusieurs éléments du texte et du contexte nous invitent à considérer sérieusement cette hypothèse.

61 Pseudo-Aristote, Problèmes, XXX, 1, dans Klibansky, Panofsky et Saxl, Saturne et la mélancolie, $58:$ : [...] les mélancoliques, pour la plupart, sont des luxurieux. »

62 Guillaume de Brescia, Practica, Modène 1510, f. 23rb : « De tertio, sciendum quod passio ista proprie accidit delitiose viventibus, solitariis, continentibus, religiosis, monialibus, otio viventibus. »

63 Thomas d'Aquin, Summa theologiae, Ia, Iiae, q. 22, a. 3, ad. 1 : « Ad primum ergo dicendum quod passio divinorum ibi dicitur affectio ad divina, et coniunctio ad ipsa per amorem. »

64 Erwin Panofsky mentionne à ce propos un texte d'Henri de Gand (Quodlibeta, II, 9), dans lequel le théologien explique que le mélancolique peut faire un bon mathématicien, en tant qu'il baigne dans l'imagination, mais ne peut penser à des objets plus hauts que ceux qui ont quelque grandeur physique ou mathématique. Autrement dit, il ne peut penser l'infini, c'est-à-dire Dieu. Cf. Klibansky, Panofsky et Saxl, Saturne et la mélancolie, 532-533. 


\section{Amour et poésie}

La réponse à nos questions se trouve peut-être dans les poèmes composés par Gentile da Foligno. Comme il l'explique lui-même, à la fin de son commentaire littéral :

Au sujet de l'amour, nombreux sont ceux qui cherchent à savoir quantité de choses : premièrement, si la femme est plus affectée par l'amour que l'homme ; ensuite par quel sens on devient amoureux, par la vue, l'ouïe ou le toucher ; et tant d'autres choses que nous ne prenons pas la peine de traiter ici, car nous en avons déjà dit beaucoup sur l'amour dans nos chansons en langue vulgaire. ${ }^{65}$

Les questions mentionnées par notre auteur sont traditionnelles, au moins depuis le commentaire de Pierre d'Espagne au Viaticum, probablement rédigé à Sienne dans les années $1250^{66}$. Quant aux autres sujets (multa alia) qu'il affirme avoir traités dans ses poèmes, il est impossible d'en mesurer l'étendue. Nulle trace, en effet, d'une canzone ou autre sonnet de Gentile da Foligno dans les nombreux Canzoniere du XIV siècle que nous avons pu consulter.

Il faut dire que les indications fournies par Gentile dans les rares allusions à ses poèmes sont plutôt minces et ne permettent pas de les identifier dans la masse des poèmes anonymes copiés dans ces immenses recueils médiévaux. Les thèmes évoqués sont si communs à l'époque, qu'on les retrouve souvent, chez plusieurs anonymes. Par exemple, alors qu'il discute des différences entre les âges de la vie en commentant le premier livre du Canon, à propos de l'adolescence il rappelle que dans une « chanson en vulgaire " (vulgari cantilena), il a écrit que l'homme commence à mourir en même temps qu'il commence à vivre ${ }^{67}$. Ailleurs, il dit avoir composé un "discours poétique en langue vulgaire » (in nostro poetico sermone vulgari) sur les cheveux blancs ${ }^{68}$. A-t-il écrit des poèmes sur l'origine divine de l'amour ? Nul ne le sait. Tentons malgré tout de situer ses propos sur l'amour à

65 Gentile da Foligno, comm. Canon, III, 1, 4, 22, Vat. Lat. 4459, f. 72va : « Circa materiam de amore multi multa querunt. Primo an mulier viro magis afficiatur amore. Secundo quo sensu quis magis amoretur, an visu, an auditu, an tactu. Et multa alia de quibus exquerere non curamus. Et iam in nostris cantilenis vulgaribus multa diximus <de $>$ amore. »

66 Voir la liste des questions contenues dans les deux versions de son commentaire dans Wack, Lovesickness in the Middle Ages, 86-87.

67 Gentile da Foligno, Comm. Canon, 1, 1, 3, 3, Venise 1520, f. 29vb : « Ad secundum dicendum quod non vocatur etas adolendi, id est perficiendi, quia addamus in potentia vivendi, quia semper plus morimur ; et nos in nostra vulgari cantilena diximus quod homo simul incipit vivere et mori [...]. » Je remercie Joël Chandelier de m'avoir indiqué ces passages.

68 Ibid., 1, 17, f. 25ra : " Canities autem cum sit languo pili, ut $5^{\circ}$ De generatione animalium capitulo $5^{\circ}$ alias capitulo $4^{\circ}$, cito advenit istis propter frigiditatem, unde deficit naturalis calor et 
l'intérieur des discussions poétiques sur l'origine divine de l'amour, thème qui a semble-t-il particulièrement intéressé les poètes-médecins italiens.

Bien qu'il s'agisse d'une thématique présente dans toute la poésie médiévale, sous diverses formes et chez de nombreux poètes, un ensemble de textes paraît spécialement pertinent pour notre enquête. Ce sont les tenzoni - l'équivalent poétique, hérité de la tenson des troubadours, des quaestiones disputatae universitaires - dont la pratique est aujourd'hui bien documentée grâce aux travaux récents sur ce qu'on a appelé la quaestio de amore ${ }^{69}$. Présente dans toute l'Italie, et notamment à Sienne ${ }^{70}$ et Pérouse ${ }^{71}$, où Gentile da Foligno a longtemps enseigné, l'une des plus célèbres tenzone sur la nature d'amour éclata au XIII ${ }^{\mathrm{e}}$ siècle, dans l'entourage de la cour de Frédéric II. Elle réunit quelques grands noms de l'école sicilienne : l'Abate di Tivoli, Jacopo Mostacci, Pier della Vigna et Giacomo Lentini $^{72}$. On a souvent insisté sur l'aspect philosophique de ces débats poétiques et sur leur influence sur Dante et Cavalcanti. Une de leurs quaestiones, par exemple, concernait la nature catégoriale de l'amour : est-ce une substance ou un accident ? Autrement dit : faut-il personnifier Amour ou en faire un accident de l'âme, une passion? Thème que l'on retrouve explicitement chez Dante et Cavalcanti. Mais la nature divine d'Amour devait aussi faire son apparition dans l'école sicilienne avant de devenir importante dans le dolce stil novo ${ }^{73}$.

Les poètes de l'école sicilienne sont en profond désaccord. Alors que l'Abate di Tivoli décrivait l'amour comme une divinité, Giacomo da Lentini refusait énergiquement cette association, tandis que Pier della Vigna en faisait quelque chose d'invisible, mais non divin, et dont l'action est pareille à celle de l'aimant, réelle

acquiritur in materia putredo aliqua sive muffulentia, et ex hoc fit canities, ut declaravimus in nostro poetico sermone vulgari quem de canitie composuimus. »

69 Pour un aperçu de ce genre littéraire, cf. C. Giunta, Versi a un destinario. Saggio sulla poesia italiana del Medioevo, Bologne 2002, en partic. 194-205.

70 On pense, par exemple, à la tenzone entre le poète siennois Cecco Angioleri et Dante par exemple.

71 Voir Poeti perugini del trecento. I. Marino Ceccoli, Cecco Nuccoli e altri rimatori in tenzone, éd. F. Mancini e L. M. Reale, Pérouse 1996.

72 Voir M. Picone, « La tenzone 'de Amore' fra Iacopo Mostacci, Pier della Vigna e il Notaio », in Id., Percorsi della lirica duecentesca, Florence 2002, 47-67.

73 La « déité d'amour » restera évoquée par Guinizzelli, Lapo et même Cavalcanti, elle est omniprésente chez Dante, et cette métaphore revient plusieurs fois sous la plume de Cino da Pistoia, l'ami de Gentile da Foligno. Par exemple, Cino da Pistoia, XLIV, 9-14 : " Così gire fa in nova sentenza / e de l'altra dipartemi spess'ore / questa gentile ad alta intelligenza, / in cui risplende deità d'Amore, / a luce a me per la somma piagenza / di quella donna, c'ha tanto valore. » Notons aussi que cette problématique n'est pas absente du Breviari d'amor de Matfré Ermangaud, le dernier des troubadours devenu franciscain, qui déplore que l'on ait fait de l'amour charnel une divinité, au détriment du Dieu véritable. 
et cependant cachée ${ }^{74}$. Plus intéressant pour notre propos, trois maestri florentins, à la fois poètes et médecins, ont continué cette tenzone au tournant du XIII ${ }^{\mathrm{e}}$ et $\mathrm{du} \mathrm{XIV}^{\mathrm{e}}$ siècle $^{75}$.

Le premier n'est autre que Pietro Torrigiano, mentionné dans les manuscrits sous le nom de Maestro Torrigiano da Firenze ${ }^{76}$. Dans les poèmes de ce médecin qui est entré dans les ordres, l'amour est appelé Dieu, au moins per similia ; car l'amour humain est un don divin ${ }^{77}$. Quant à la cause de l'amour en l'homme, les poèmes semblent décrire une réalité quelque peu différente de ce qu'on peut lire dans son commentaire au Tegni, où l'amour est considéré comme une simple émotion, qui engendre une certaine joie (gaudium), au même titre que l'espoir ${ }^{78}$. Dans le sonnet Chi non sapesse ben la veritate, il explique que l'amour est un désir de l'âme, un effort de la pensée qui maintient l'esprit en joie et le guide, vers Dieu, précisément parce que Dieu en est la cause ${ }^{79}$. La joie dont il est désormais question est plus qu'une simple émotion et donne en quelque sorte un contenu moral à l'amour.

Cette vision d'un amour divin est explicitement refusée par Maestro Francesco da Firenze, lui aussi médecin ${ }^{80}$. De fait, explique-t-il, « nombreux sont ceux qui appellent l'amour déité » (Molti l'amore apellano dietate), en raison de

74 Cf. W. Pagani, Repertorio tematico della scuola poetica siciliana, Bari 1968, 7-12.

75 Certains ont même proposé de rattacher directement ces sonnets à la tenzone entre l'Abate di Tivoli, Jacopo Mostacci, Pier della Vigna et Giacomo Lentini. Par exemple S. Santangelo, Le tenzoni poetiche nella letteratura italiana delle origini, Genève 1928. Sergio Lubello y voit quant à lui une autre dispute, dans un autre contexte. Cf. I poeti della scuola siciliana, vol. III : Poeti siculo-toscani, R. Coluccia (éd.), Milan 2008, 417.

76 Sur l'identité du poète, cf. F. Catenazzi, « Per maestro Torrigiano da Firenze », Rivista di letteratura italiana VI (1988), 265-273 et S. Gentili, L'uomo aristotelico : alle origini della letteratura italiana, Rome 2005, 201-216.

77 Maestro Torrigiano da Firenze, 45, 1, éd. S. Lubello, dans I poeti della scuola siciliana, vol. III, 417 : « Chi non sapesse ben la veritate / come l'Amor sia deo, ora lo 'ntenda : / di quante cose ne son nominate / per questo nome « deo », primera aprenda. / Dette a Natura Deo la maestate, / e da la forma par che 'l nome penda, / e tal per grazia e tal per potestate / si chiama Dio per simile vicenda. »

78 Pietro Torrigiano, Plusquam commentum in parvam Galeni artem, Venise 1557, f.104r.

79 Maestro Torrigiano da Firenze, 45, 1, éd. S. Lubello, dans I poeti della scuola siciliana, vol. III, 45, 2 (420-421) : « Nè volontier lo dico, nè lo taccio, / qual cosa sia l'amor che dio s'apella [...] / L'amor, di cui la gente canta e grida, / è uno disio de l'arma, che pensosa / la tiene in gioia d'amore, ove si fida ; / e quest'è de l'amor la propia cosa, / che pur al suo timon l'arma si guida: / per ciò ciascuno amante dio lo cosa. »

80 On ne sait rien de l'identité de ce personnage, mais, comme le suggère Sergio Lubello, le titre de maître, ainsi que les thèmes qu'il traite dans ses poèmes et l'attention particulière qu'il porte au vocabulaire technique, sont des éléments qui semblent indiquer une proximité certaine avec les milieux universitaires et plus particulièrement avec la médecine. Cf. ibid., 362, 393 et 417. 
sa puissance et de sa souveraineté sur les actions humaines, mais ce n'est pas la vérité, comme le prétendait Pietro Torrigiano : C’amor sia deo non è la veritate. Ceux qui décrivent ainsi l'amour le font simplement par ignorance, alors que ce ne devrait être qu'une métaphore : parce qu'il est invisible (perch'om visibolmente nol comprende), ils en font quelque chose de divin, mais c'est là un raisonnement fallacieux. On retrouve donc chez lui le motif déjà utilisé par Pier della Vigna : tout ce qui est invisible n'est pas divin. La réponse finale de Maestro Francesco est intéressante. Sur le plan théologique d'abord, il oppose à Torrigiano un argument traditionnellement utilisé dans les problèmes de théodicée : si l'amour était un don divin - et donc un bien - pourquoi nous ferait-il tant souffrir ? Comme il l'écrit : che Deo per bene già male non rende. Autrement dit, si c'est un mal, physique et moral, il faut que ce soit l'effet d'un défaut du corps ou d'un acte volontaire, non un don divin. Sur le plan psycho-physique, Maestro Francesco préfère donc expliquer la puissance de l'amour par les effets de l'image et de la pensée, dont les mécanismes sont certes occultes, mais ne manquent pas d'avoir des effets visibles sur le corps ${ }^{81}$. Quelle est donc « la vérité » sur le mal d'amour? C'est une passion qui naît de la vision d'une belle forme, qui devient progressivement l'objet d'une pensée inquiète, sans repos, et qui, une fois le cœur atteint, provoque le désir malheureux de l'homme passionné ${ }^{82}$.

Quant au troisième homme, Maestro Rinuccino, médecin florentin actif dans les années 1290 et mort avant $1299^{83}$, il formulait l'argument théologique d'une autre manière, en partant cette fois du caractère injuste d'Amour, caractère contradictoire avec l'idée d'un amour providentiel. Si l'amour était divin, s’il gouvernait le cœur des hommes, pourquoi cette divinité n'a-t-elle pas fait en sorte que l'amour soit toujours réciproque entre les amants et qu'il les rende

81 On retrouve l'association du thème de l'invisibilité et celui de l'impossibilité théologique d'une origine divine de l'amour dans une tenzone anonyme (I poeti della scuola siciliana, vol. II, 1015-1017) : « Amor nonn-è, se non come cred'eo, / cosa ch'om possa veder né tocare, / ma sono molti che l'apellan deo : / sono inganati ed ànno van pensare, / ca se deo fosse, non facera reo: / ca 'n deïtate è tutto degno afare. "

82 Maestro Francesco da Firenze, 42, 8, éd. S. Lubello, dans I poeti della scuola siciliana, vol. III , 394 : « Ma che sia amore, eo dicerò lo vero : / quand'om diven solicito e pensoso / vegendo uno bel viso e piacentero, / à mantenente amore in cor rinchiuso ; / c'amore è uno continovo pensero / di quella cosa ond'omo è disïoso. » On reconnaît dans l'expression « solicito e pensoso » une référence possible à la définition avicennienne de la passion amoureuse.

83 Pour sa biographie, voir F. Catenazzi, «Contributo alla conoscenza di Maestro Rinuccino, poeta fiorentino del secolo XIII », Studi e problemi di critica testuale XX (1980), 97-118; et l'introduction de S. Carrai, I sonetti di Maestro Rinuccino da Firenze, Florence 1981, 11-19. 
parfaitement heureux ${ }^{84}$ ? Car ce n'est pas le cas, à l'évidence, et l'amour non réciproque représente une forme d'injustice inadmissible s'il est le fruit d'un décret divin. Ce qu'il dénonce, semble-t-il, comme Maestro Francesco, c'est la confusion entre l'amour intellectuel de Dieu, compris comme vertu théologale (la caritas), et l'amour dont s'occupent les poètes et les médecins, c'est-àdire celui qui prend sa source dans la rencontre de deux personnes ou parfois dans un simple fantasme chez un individu. Cet amour-là, qui provoque toutes les souffrances si souvent décrites par les poètes, et auquel les médecins sont confrontés concrètement, ne saurait être réduit à la forme supérieure d'amour évoquée par Torrigiano. Il faudrait, à tout le moins, une forme de conversion d'un amour vers l'autre, comme l'explique Dante dans la Vita nova, par exemple. Mais Francesco et Rinuccino semblent considérer que cet amour ne constitue aucunement l'objet du poète et du médecin. Ces derniers doivent s'en tenir à la passion qui dévore les amants.

Les échanges entre Dante et Cavalcanti ont certainement contribué à relancer ce débat au XIV ${ }^{\mathrm{e}}$ siècle. Le premier, en particulier dans la Vita nova, mais aussi dans la Commedia, ne décrivait-il pas la première forme d'amour dans les termes de la médecine galénique, pour mieux montrer ensuite comment cet amour physique devait se transformer en amour de la sagesse, puis, en dernière instance, en amour de Dieu ? Guido Cavalcanti, au contraire, ne résumait-il pas l'amour à ses manifestations physiques, entraînées par la contemplation d'une image, qui se transformait en passion ? Si l'arrière-plan du commentaire de Gentile da Foligno était celui-ci, ne peut-on imaginer qu'il a tenté de répondre en médecin à la quaestio de amore, comme l'avait fait avant lui Dino del Garbo en commentant la chanson Donna me prega de Cavalcanti ? Après tout, Gentile da Foligno a connu Dino del Garbo à Sienne lorsqu'ils enseignaient tous deux la médecine dans cette université (entre 1321 et 1323) et il a fréquenté Cino da Pistoia, l'ami de Dante, lorsqu'ils enseignaient aussi dans la même université, à Sienne et à Pérouse. Il serait donc tentant de voir dans cette brève explication de la passio herotica l'évocation, certes vague, du débat poétique rappelé ci-dessus, puisqu'il opposa des poètes-médecins aux profils assez proches de celui de Gentile da Foligno. Si tel était le cas, Gentile da Foligno voulait peut-être dire que cette passion n'est pas

84 Maestro Rinuccino da Firenze, Sonetto, VIIIa, éd. S. Carrai, dans I sonetti di Maestro Rinuccino, 74-75 : «S’Amor fosse formato in dietate, / sì come àn detti manti trovadori, / a ver’ li amanti avesse potestate / di meter nove voglie ne li cori, / un prego gli faria con umiltate : / c'ogn'om d'amor ferisse, chi n'è fori, / a ciò che si cangiasse l'amistate / che spesse fiate è posto in due colori. // Questo saria un guisto segnorag[g]io : / che l'om c'amasse fosse tanto amato / quant'ama, sì che stesse in par gradag[g]io, / e che l'amor crescesse di parag[g]io, / sì che d'amore fosse l'om cangiato / e par fossor gli amori nel corag[g]io. » 
divine au sens d'un don de Dieu, mais procurerait une capacité d'abstraction des préoccupations mondaines propice à l'inspiration poétique, sans être le strict équivalent de l'amour de Dieu.

En tout cas, dans la suite du texte, Gentile da Foligno défend une position très différente de celle de Pietro Torrigiano ${ }^{85}$. Laissant de côté le caractère divin de la maladie d'amour, il insiste désormais, plus que les autres médecins, sur l'aspect mental de cette affection. Pour ce faire, Gentile da Foligno distingue assez nettement la simple mélancolie et le mal d'amour authentique. Ni purement corporelle ni tout à fait divine, la passion amoureuse a davantage trait à l'image et au désir.

\section{La mélancolie, le désir et les puissances de l'âme}

Contrairement à la simple mélancolie, il n’y aurait dans la passion amoureuse aucun lien nécessaire entre un défaut au niveau des humeurs - de la bile noire en particulier - et le mauvais fonctionnement de l'âme, que ce soit au niveau de l'imagination, de la mémoire ou de la faculté estimative. Un tel lien causal peut certes exister, mais il est contingent :

En second lieu, il convient d'observer qu'Avicenne dit que cette passion est semblable à la mélancolie, parce que cette passion se rapporte à la mélancolie mais diffère d'elle, comme cela apparaît dans l'intention [du texte]. Elle s'y rapporte, parce que dans les deux cas on constate un dommage dans la [fonction] cogitative, accompagné de beaucoup d'inquiétude. Mais elle en diffère, parce que la mélancolie est nécessairement produite à partir d'un simple défaut au niveau de l'humeur ou de la complexion, c'est-à-dire à partir de dispositions corporelles malades chez cet individu. Quant à l'amour, il se produit par la seule force du jugement de [la faculté] estimative, relativement à tel individu qu'elle peut juger, bien que cela puisse aussi aller de pair avec un défaut de l'humeur ou même se produire par un défaut d'humeur ${ }^{86}$.

85 Gentile da Foligno rédigea d'ailleurs un petit commentaire au Tegni pour corriger les erreurs de Pietro Torrigiano. Le texte est édité à la suite du commentaire de Torrigiano dans l'édition de 1557. Voir les remarques de P. -G. Ottosson, Scholastic Medicine and Philosophy. A Study of the Commentaries on Galen's Tegni (ca.1300-1450), Naples 1984, 49.

86 Gentile da Foligno, comm. Canon, III, 1, 4, 22, Vat. Lat. 4459, f. 72rb : « Considerandum secundo quod Avicenna dicit quod hec passio est similis melancolie, quia ut ex eius intentione apparet, ista passio convenit cum melancolia et differt ab ea. Convenit, quia in utraque est lesio cogitative cum multa sollicitudine. Sed differt, quia melancolia fit necessario a vitio humorali sive complexionali puro, que sunt dispositiones corporales egre respectu illius individui ; sed amor fit sola vehementia extimationis respectu huius individui extimabilis, licet etiam possit coniungi vitium humoris, vel etiam a vitio humoris fieri. » 
C'est donc parfois le mauvais fonctionnement de l'âme qui engendre une modification des humeurs et non l'inverse. Contrairement à la mélancolie, l'image peut agir seule et être efficace sans dérèglement préalable du corps. L'interprétation de Gentile da Foligno marque ainsi un changement notable par rapport à la tendance forte à son époque de rapprocher mal d'amour et mélancolie. Le Viaticum, par exemple, mentionnait l'excès de bile noire comme une cause de la passion amoureuse, tandis que d'autres auteurs, comme Arnaud de Villeneuve, $\mathrm{y}$ ajoutaient la colère jaune ${ }^{87}$. De manière générale, même si tous les médecins reconnaissaient le rôle central de la pensée et de l'imagination dans la passion amoureuse, les causes corporelles étaient très souvent mises en avant aux XIII ${ }^{\mathrm{e}}$ et $\mathrm{XIV}^{\mathrm{e}}$ siècles. Gentile da Foligno préfère insister sur le dysfonctionnement interne de l'âme. Autrement dit, la passion amoureuse n'est pas d'abord un problème corporel, mais bien une maladie de l'âme.

Reste à savoir à quelle fonction de l'âme on doit l'attribuer. Au sens strict, écrit Gentile da Foligno, l'amor hereos est une maladie ou un dérèglement de l'appétit (appetitus), c'est-à-dire du désir sensible, par lequel un homme désire une femme ${ }^{88}$. C'est ce qui distingue l'amoureux transi du simple mélancolique, maladie dont les effets peuvent être le fruit d'une maladie du cerveau et des fonctions cognitives de l'âme, sans que le désir soit affecté. Certes, précise-t-il, la passion amoureuse touche la quasi-totalité des fonctions cognitives et motrices de l'âme sensible, puisqu'elles sont toutes affectées par l'intensité (intensio) de l'appréhension interne de la forme de l'être aimé. La faculté estimative de l'âme, logée dans le ventricule médian du cerveau, est particulièrement atteinte par cette intensité. L'imagination et la mémoire sont altérées, mais la faculté estimative est corrompue, puisqu'elle n'opère plus sa fonction ${ }^{89}$. C'est pourquoi, même si la

87 Voir les commentaires de Danièlle Jacquart dans Jacquart et Thomasset, " L'amour héroïque », 156-157. Comme elle l'écrit (157) : « D’autres médecins tireront plus radicalement l'amour vers la mélancolie : Jean de Gaddesden et Jean de Tournemire le définiront comme une forme de celle-ci. »

88 Gentile da Foligno, comm. Canon, III, 1, 4, 22, Vat. Lat. 4459, f. 72rb : « Dubitatur cui virtuti attribuatur amor. Et dicendum quod formaliter virtuti appetitive. Ratio est, quia amor formaliter non videtur esse aliud nisi appetitus sive dispositio illum consequens, unde homo appetit aliquam dominam ; ergo videtur <esse> passio virtutis appetitive, scilicet concupiscitive. »

89 Gentile da Foligno, comm. Canon, III, 1, 4, 22, Vat. Lat. 4459, f. 72rb : « Dico etiam quod cuilibet virtuti cognoscitive intrinsece debet quodam modo attribui. Et ratio est, quia cuiuslibet virtutis operatio concurrit. Primo enim concurrit sensata forma, que apprehenditur ab imaginativa et affert illam aliquis particularis sensus. Secundo concurrit placibilitas iudicata per extimativam. Tertio concurrit continuatio iudicii placibilitatis ex continua reportatione per memorativam. Ex quibus vigoratis et unitis consurgit appetitus coniungendi se cum illa muliere quam homo diligit ; et quia assecutio fit cum membris generativis, ideo fit titillatio circa illam. Ex quibus apparet 
passion amoureuse affecte formellement le désir, la fonction estimative joue un rôle particulièrement important dans le processus qui l'engendre. Cette fonction cognitive porte en effet sur l'objet senti par les sens externes et forme à leur propos une sorte de pré-jugement, proche de l'instinct animal, par lequel l'individu juge que l'objet de connaissance est plaisant ou non, sympathique ou effrayant, ce qui le conduit à poursuivre ou à fuir l'objet de cette pensée. En d'autres termes, cette appréhension de la forme dans l'âme va motiver ou non le désir. Une fois activé, ce désir, fondé sur l'image déformée par l'estimative et l'imagination, peut croître jusqu'à engendrer un cercle vicieux : le désir accroît la pensée et la pensée accroît le désir.

On retrouve donc chez Gentile da Foligno la même analyse que celle de Dino del Garbo dans son commentaire de la chanson Donna me prega de Guido Cavalcanti. Commentant les vers non è vertute, ma da quella vène, le médecin expliquait que l'amour ne pouvait être qu'une passion, c'est-à-dire un accident d'une puissance de l'âme. C'est une passion de l'appétit sensible, précise Dino del Garbo, comme le sont les autres passions, comme la colère, la tristesse ou la peur.

Car l'amour est une certaine passion du désir sensible, parce que le désir découle de l'appréhension de la forme d'une chose par les sens, d'abord extérieurs, puis par les puissances sensibles intérieures, comme on l'a dit plus haut : c'est pourquoi dans l'amour concourent deux puissances sensibles, la connaissante et la désirante, parce que tout désir qui se trouve en nous découle d'une connaissance. Et, bien que dans l'amour concoure l'opération de la puissance cognitive sensible, l'amour ne se trouve pourtant pas dans la puissance cognitive sensible comme dans son sujet ; et il en est ainsi parce que dans la connaissance il y a seulement un mouvement des choses vers l'âme, tandis que dans le désir il y a un mouvement de l'âme vers les choses ${ }^{90}$.

Cette explication est sensiblement différente de ce que l'on pouvait lire au siècle précédent chez un Gérard de Berry par exemple. Dans son commentaire au Via-

quod in amore est lesio imaginative et memorative per intensionem actus ; sed extimativa est lesa per corruptionem, quia iudicat quandoque pulchrum quod est sedum, et per intensionem, quia continua sua actio est extimatio de pulchritudine et placibilitatte illius. Et omnia alia extimabilia quasi obmittit. »

90 Dino del Garbo, Scriptum super cantilena Guidonis Cavalcantibus, dans Fenzi, La Canzone d'amore di Guido Cavalcanti, 106-108 : « Nam amor est passio quedam appetitus, qui appetitus consequitur formam rei apprehense per sensum primo exteriorem et deinde per virtutes sensitivas interiores, ut dictum est supra : unde in amore concurrit duplex potentia sensitiva, scilicet cognoscitiva et appetitiva, quia omnis appetitus qui est in nobis insequitur cognitionem. Et, licet in amore concurrat operatio potentie cognoscitive sensitive, tamen amor non habet esse proprie ut in subiecto in potentia sensitiva cognoscitiva, sed habet in appetitiva, sicut omnis alia passio, et hoc ideo est quia in cognitione sola est motus rerum ad animam, sed in appetitu est motus anime ad res ». 
ticum, il définissait la passion amoureuse comme une maladie du cerveau, et plus particulièrement de la faculté estimative, et décrivait les mécanismes qui conduisent à cette passion en termes quasi matérialistes, selon la séquence suivante $^{91}$ : une forme est sentie par un ou plusieurs sens externes ; la faculté estimative appréhende cette forme comme bonne ou mauvaise, en attribuant parfois des propriétés à cet objet qui n'existent pas ; elle commande ensuite à l'imagination, qui va se focaliser sur cette forme ; l'imagination, elle, commande le désir. L'imagination se fixe sur cet objet en raison du déséquilibre de la complexion du ventricule du cerveau où elle se trouve. De même, le défaut de la fonction estimative viendrait de la concentration de spiritus et de chaleur naturelle dans la partie du cerveau qui l'accueille. Gentile da Foligno ne nie aucunement l'existence d'une causalité naturelle dans le phénomène amoureux, mais il insiste fortement sur la primauté de l'image et de sa saisie par les différentes fonctions cognitives de l'âme jusqu'au désir. Les défauts de complexion ou d'humeur sont plutôt le résultat de l'intensité des pensées, et non l'inverse. Une telle insistance sur le rôle de la pensée se comprend mieux dès lors que l'on se tourne vers la question des modalités de l'action de l'âme sur le corps.

\section{Le pouvoir de l'âme sur le corps}

Dans le chapitre sur l'amour, Avicenne se contente d'affirmer, au détour d'une phrase, que cet état pathologique illustre le principe général selon lequel la nature « obéit » à la pensée ${ }^{92}$. Comment le corps obéit-il aux injonctions de l'âme, qu'elles soient volontaires ou involontaires ? Dans le dualisme avicennien, l'âme et le corps sont considérés comme deux entités distinctes, agissant réciproquement l'une sur l'autre, mais sur un mode distinct de celui des corps entre eux ou des idées entre elles. Pour cette raison, Avicenne défend une idée pour le moins surprenante : l'âme peut agir sur un corps qui n'est pas le sien, comme en témoigneraient certains phénomènes d'action à distance ${ }^{93}$. Cet argument implicite pour le dualisme - si l'âme peut agir sur un corps distant, sans contact, alors elle peut a fortiori agir sur le corps qui est le sien, tout en étant ontologiquement distincte de lui - a fait l'objet d'un intense débat parmi les médecins

91 Gérard de Bérry, Glosulae super Viaticum, dans Wack, Lovesickness in the Middle Ages, 198-200.

92 Avicenne, Canon, III, 1, 4, 22, f. 90vb.

93 Avicenne, Liber de anima seu Sextus de naturalibus, IV, 4, éd. S. Van Riet, vol. II, LouvainLeyde 1968, 54-67. 
italiens, lesquels critiquèrent unanimement la possibilité d'une action de l'âme à distance ${ }^{94}$. Il restait cependant à expliquer certains passages du Canon dans lesquels Avicenne reprenait ce thème de l'action de l'âme sur le corps. Il affirmait, par exemple, que « les jugements [de la faculté estimative de l'âme] eux-mêmes meuvent les humeurs » (ipse aestimationes movent humores), ou encore que ces jugements peuvent modifier la complexion du corps ${ }^{95}$. À part Taddeo Alderotti, qui semble encore sous le charme des thèses avicenniennes, Antoine de Parme, Pietro d'Abano et Dino del Garbo, pour ne citer qu'eux, tentèrent d'expliquer l'action de l'âme sur le corps à partir de l'efficacité des images, depuis la sensation jusqu'aux fonctions les plus hautes de l'âme animale comme la faculté estimative, et des émotions que ces images suscitent, véritables déclencheurs, la plupart du temps, des effets physiques constatés. Gentile da Foligno s’inscrit clairement dans cette tradition.

Dans le chapitre sur l'amour, Avicenne se contente donc de rappeler que la nature obéit aux pensées (significavimus obedientiam nature meditationibus). Gentile, lui, voit tout de suite la référence implicite à la célèbre thèse du De anima d'Avicenne : lorsque l'âme est bien disposée, le corps lui obéit, immédiatement, de manière quasi miraculeuse ${ }^{96}$. Mais il ne donne guère d'explication. C'est dans son commentaire à la première fen du livre I que l'on trouve plus de détails.

Une forme (species) dans l'âme peut-elle être la cause d'un changement réel $^{97}$ ? Comme la plupart de ses prédécesseurs, Gentile da Foligno commence par évacuer la thèse avicennienne de l'action de l'âme sur un corps distant. Sans véritablement donner d'argument, il se contente d'affirmer ne pas vouloir discuter ici la vérité de cette affirmation ${ }^{98}$. Les enjeux philosophiques et théologiques

94 Nous avons déjà étudié en détail cette querelle dans A. Robert, « Dino del Garbo et le pouvoir de l'imagination sur le corps ", Archives d'histoire littéraire et doctrinale du Moyen Âge 81 (2014), 139-195.

95 Avicenne, Canon, I, 2, 2, 1, 14, f. 114ra.

96 Gentile da Foligno, comm. Canon, III, 1, 4, 22, Vat. Lat. 4459, f. 72rb : « Amplius, hic actende mentem Avicenne quanta est obedientia corporis extimationi et huius sententie est sexto De naturalibus, particula 4, et De animalibus $7^{\circ}$, ubi dicit quod quando gallina vincit gallum in pugna erigitur quasi esset gallus et elevat suam caudam sicut gallum ; et aliquando nascitur illi cornu in crure quasi gallo ; et in hoc percipi potest obedientia nature cogitationibus anime, etc. »

97 Gentile da Foligno, comm. Canon, I, 1, 4, 2, f. 53ra : «Dubitatur communiter utrum species in anima sit causa realis transmutationis.»

98 Ibid.: «Et dicamus primo opinio fuisse Avicenna 6 De naturalibus particula 4 capitulo 4 et Algazelis ultimo sue Metaphysice, quod anima unius hominis sive imaginatio imprimit in aliud corpus extra se, ut cum anima alicuius fuerit nobilis, constans, ex nobilibus principiis, obedient ei elementa et res mundane transmutabiles, ut eius voluptate (sic, pro voluntate) proveniat pluvia et lapides, fiant panes et alii effectus provenient in homines quos effectus vocamus fascina- 
d'un tel problème dépassent largement le périmètre de l'expertise médicale et, surtout, ce n'est pas un problème qui l'intéresse particulièrement ${ }^{99}$. Il ne traite donc que de l'action de l'âme sur le corps du sujet pensant ou imaginant.

Bien que sa position ne soit guère originale et reprenne en partie les arguments d'Antoine de Parme et Dino del Garbo, elle offre cependant une synthèse des débats antérieurs fort utile pour comprendre comment, dans le phénomène amoureux, la simple pensée d'une forme (species) dans l'âme peut produire des effets délétères sur le corps. Notre médecin commence par rappeler que les plus grandes autorités philosophiques et médicales s'accordent sur l'existence de tels effets de la représentation mentale sur le corps. Le Pseudo-Aristote, par exemple, décrit dans ses Problèmes les mécanismes par lesquels nous nous mettons à tousser ou bailler à la simple vue de quelqu'un toussant ou baillant, ou même simplement en l'imaginant. Galien, dans son traité De motibus liquidis, explique pourquoi nous frémissons à la simple vue d'une lame acérée. Enfin, Avicenne écrit, dans son De anima, que nous ne pouvons pas traverser un pont suspendu au-dessus d'une rivière si nous imaginons la chute ${ }^{100}$. Il ajoute enfin qu'il va de soi que « l'imagination d'une femme lascive est la cause d'un chatouillement des

tiones. Utrum autem hec opinio fuit vera omittamus. » Ce passage est une citation transformée du De anima d'Avicenne, 65.

99 D'autres médecins italiens, comme Antoine de Parme, Pietro d’Abano, Dino del Garbo, Iacopo da Forli ou Ugo Benzi, ont abordé cette question de manière très précise en commentant le Canon d'Avicenne. Cf. Robert, « Dino del Garbo et le pouvoir de l'imagination ». Pour une histoire de ces discussions, cf. A. Godet, « Num was ist die Imagination anderst als ein Somn im Menschen. »Studien zu einem Zentralbegriff des magischen Denkens (thèse de doctorat), Basel 1980 ; P. Zambelli, «L'immaginazione e il suo potere. Da al-Kindi, al-Farabi e Avicenna al Medioevo latino e al Rinascimento », dans Orientalische Kultur und europäisches Mittelalter, Berlin-New York 1985, 188-206 ; A. Boureau, « Miracle, volonté et imagination : la mutation scolastique (1270-1320) », Actes des congrès de la Société des historiens médiévistes de l'Enseignement supérieur public, $25^{\mathrm{e}}$ congrès, Orléans, 1994, Paris 1995, 159-172 ; T. Griffero, Immagini attive. Breve storia dell'immaginazione transitiva, Florence 2003 ; B. Delaurenti, La puissance des mots. «Virtus verborum ». Débats doctrinaux sur le pouvoir des incantations au Moyen Âge, Paris 2007.

100 Gentile da Foligno, comm. Canon, I, 1, 4, 2, f. 53ra : "Secundo dicamus opinio fuisse omnium vere philosophantium quod species in anima sive imaginatio sit causa realis motus in corpore eodem. Hoc apparet ex pensatis (sic pro sensatis) ; primo quia vides (sic pro videns) alio comedente acetosa, dentes imaginantis quandoque stupefiunt, et alio oscitante nos oscitamus, et alio mingente excitamur ad mingendum ; et hec omnia concedit Aristoteles Problemata 7, problemum primo 5 et problemum 8 ; et Galenus in libro De motibus liquidis : aliquo (sic pro aliquando) etiam acuentem ferram vel incidentem pumicem vel lapidem moliri horrescimus ; concedit his similia Aristoteles Problemata 7, problemum 8. Videmus etiam quod super trabe posita super flumen non possumus transire, ut Avicenna 6 De naturalibus particula 4 capitulo 4, nec etiam supra pinnaculo alti montis, ut Galenus concedit in libro De motibus liquidis capitulo 7 secunde distinctionis. » 
testicules $»^{101}$, là où Avicenne se contentait de dire que parfois un membre se tend par le seul effet de l'imagination ${ }^{102}$. Une fois les effets admis, il reste à déterminer les modalités de cette action psycho-physique.

Gentile da Foligno distingue alors deux voies possibles. Dans certains cas, explique-t-il, il faut reconnaître l'existence d'une communication entre l'agent et le patient, c'est-à-dire un rapport quasi matériel, comme l'impose la théorie aristotélicienne de l'altération exposée dans le livre VII de la Physique d'Aristote ${ }^{103}$. Cette communication, à l'origine d'une forme d'altération, se situerait au niveau de l'image elle-même, comme le suggère Aristote lui-même dans son traité Sur le mouvement des animaux ${ }^{104}$. Gentile da Foligno poursuit en émettant l'hypothèse selon laquelle l'image dans l'âme retient quelque chose de sa cause, dont elle est la représentation, à la manière de la semence masculine qui transmet à l'enfant certains caractères du père. C'est là, dit-il, la position du Pietro d'Abano, pour qui « la forme (species) qui est dans l'âme, qu'elle soit chaude ou froide, ou encore aigre, ou de quelque autre nature, peut donc d'une certaine manière conserver la puissance des choses en dehors de l'âme ${ }^{105}$.

Gentile est toutefois conscient des limites de ce modèle qui attribue à l'image elle-même des propriétés physiques. Pourquoi de tels effets ne se produisent pas dans un miroir lorsqu'une image s'y reflète ? Comment se fait-il qu'une même image puisse produire des effets contraires selon les circonstances qui entourent son appréhension ? Un premier élément de réponse consiste à ajouter aux pouvoirs de l'image l'intervention de certaines dispositions du côté du patient : la forme ou species agit sur l'âme, puis sur le corps, lorsqu'ils sont disposés à recevoir cette action. Plus précisément, l'âme se dispose elle-même activement à pâtir sous l'effet de ces images. Une première disposition est en effet assurée par l'action de la faculté estimative « par laquelle l'homme désire et veut poursuivre ou fuir [quelque chose], et par laquelle il est disposé à certaines émotions (accidentia animae), comme la colère, la tristesse, et ainsi de suite ${ }^{106}$. La species est d'abord reçue par l'entremise des sens, avant d'être entreposée dans l'ima-

101 Ibid. : «Imaginatio autem lascivientis mulieris est causa titillationis genitalium. » 102 Avicenne, Liber de anima seu Sextus de naturalibus, IV, 4, 62.

103 Pour une présentation claire de ces requisits, cf. S. Maso, C. Natali, et G. Seel (éds.), Reading Aristotle's Physics VII, 3. What is alteration?, Las Vegas-Zurich-Athens 2012.

104 Aristote, De motu animalium, (translatio Guilelmi de Moerbeka), éd. P. De Leemans, Turnhout 2011 (Aristoteles Latinus, 17/2.2-3), 7, 701 b 16-22, (59), 1. 228-233.

105 Gentile da Foligno, comm. Canon, I, 1, 4, 2, f. 53ra : «Et voluit hoc Conciliator : in specie ergo que est in anima calidi vel frigidi vel acetosi et sic de aliis potest aliquo modo salvari virtus rerum extra."

106 Ibid. : « Una est dispositio sequens actum estimativum qua homo appetit et vult prosequi aut fugere et qua disponitur accidentibus anime, scilicet ira, tristitia et huiusmodi. » 
gination et filtrée, en quelque sorte, par la faculté estimative. Après cela, selon les modalités d'appréhension de l'estimative, un jugement se forme dans cette faculté, ce qui entraîne la formation de certains désirs et certaines émotions. Ce sont donc in fine "les accidents de l'âme ", c'est-à-dire les passions, qui sont responsables des effets physiques de la pensée, parce que ces accidents entraînent naturellement un mouvement des esprits (spiritus), centrifuge ou centripète, dans tout le corps.

Bien que de tels mécanismes nous soient cachés, il ne saurait y avoir d'autre cause aux effets de la colère que les mouvements centrifuges de spiritus initiés par cette émotion primordiale. Dans ce cas, ce n'est pas l'image qui meut immédiatement le corps, comme certains passages du De anima d'Avicenne et du De motu animalium d'Aristote pouvaient le suggérer. C'est grâce à des mécanismes de réaction aux images, inscrits dans notre nature sous forme de dispositions, que certaines pensées deviennent efficaces. Quant à savoir ce qui meut véritablement le spiritus dans le corps, c'est là, nous dit Gentile, une affaire de subordination naturelle des facultés de l'âme et du corps. Notre nature est ainsi faite : les esprits qui circulent dans le corps sont subordonnés à l'âme animale, c'est-à-dire, précise-t-il, aux puissances cognitives et au désir. Parfois ce sont les puissances naturelles (virtutes naturales), sises dans certains membres du corps, qui mettent les esprits en mouvement, mais c'est toujours sous les ordres de la connaissance et du désir. Ainsi, malgré un effort pour expliquer l'obéissance du corps à l'âme, il y a comme un point d'arrêt indépassable dans l'explication scientifique, au-delà duquel, en tout cas, le médecin ne s'aventure pas.

Gentile da Foligno en vient donc à la seconde modalité d'action de la représentation sur le corps. Parfois, explique-t-il, le corps peut être affecté par la seule appréhension d'une forme dans l'âme, c'est-à-dire sans acte de la faculté estimative et sans l'intervention des passions ${ }^{107}$. Le cas emblématique d'une telle action est décrit par Avicenne dans le Canon : il suffit parfois d'imaginer quelque chose au moment du coït pour que l'enfant à naître s'assimile à ce contenu imaginatif ${ }^{108}$.

107 Gentile da Foligno, comm. Canon, I, 1, 4, 2, f. 53ra : « Alia dispositio in anima cognoscitiva per speciem apprehensam est sola speciei imago et fixus intuitus, quamvis non sequantur aliqua accidentia anime, sicut cum simpliciter imaginor album vel nigrum absque aliquo appetitu fuge vel prosecutionis, et ex tali imaginatione sola et fixa movetur quandoque corpus realiter, sicut cum assimilatur homo natus ei qui fuit imaginatus in hora coitus ; et causa istius alterationis vel est virtus speciei quam reservat a re a qua species deciditur vel est ordo naturalis virtutum subiectarum et subordinatarum anime naturali, scilicet cognoscitive, ut tali specie fixa existente in anima spiritus sic moveantur et sic etiam humores ; et in hoc cadit diversitas secundum diversitatem hominum in specialibus dispositionibus. "

108 Avicenne, Canon, I, 2, 2, 1, 14, Venise 1520, t. I, f. 114ra. 
Cela peut s'expliquer par l'idée aristotélicienne d'une conservation du pouvoir du représenté dans la représentant ou simplement par la subordination des facultés inférieures à l'âme connaissante et désirante. Et l'on retrouve ici la même référence au De anima d'Avicenne que dans le chapitre sur l'amour : l'exemple d'une poule combattant un coq en se prenant elle-même pour un coq. La force acquise par la poule dans le combat lui vient de l'imagination, que même une gallinacée possède. Gentile conclut simplement : « ainsi, dans cet exemple, on peut percevoir l'obéissance de la nature à la pensée de l'âme, ainsi que dans le troisième livre du Canon dans le chapitre sur l'amour ${ }^{109}$.

Parfois donc, l'image agirait par elle-même chez l'amoureux, c'est-à-dire par ses propriétés intrinsèques, représentationnelles ou matérielles, en se substituant à l'être aimé ; parfois, au contraire, les effets de l'amour découleraient du jugement de la faculté estimative, laquelle permet l'apparition du désir et de certaines passions ou émotions, lesquelles entraînent immédiatement des mouvements de spiritus et de chaleur naturelle dans tout le corps.

Gentile da Foligno ne tranche pas vraiment pour l'une ou l'autre de ces deux solutions, au demeurant peu satisfaisantes. On remarque cependant un point intéressant. Un même contenu mental peut avoir des effets différents selon les dispositions de chaque individu et selon les circonstances qui entourent ces pensées. Gentile donne ici un exemple trivial : imaginer quelque chose d'aigre peut procurer de la joie chez un individu et engendrer ainsi un réchauffement du corps, mais peut plonger un autre dans la tristesse - et donc engendrer un refroidissement du corps - tandis qu'un troisième n'éprouvera aucune émotion particulière. En appliquant cet exemple au cas de l'amour, cela donnerait : imaginer ou se souvenir d'une même personne aura des effets différents selon les sujets de ces imaginations et de ces souvenirs. Cela dépendra du jugement de la faculté estimative sur ce même contenu et aussi, par voie de conséquences, des émotions et du désir qu'un tel jugement engendre.

On comprend mieux dès lors comment soigner cette maladie. Puisque l'image est au cœur du mécanisme de la passion, il faut divertir le malade, détourner son imagination vers d'autres objets. Il faut en outre susciter en lui de nouvelles émotions. En effet, lorsque Gentile da Foligno aborde la cure de la passion amoureuse, il se contente de rappeler qu'Avicenne propose trois sortes de soins : les

109 Gentile da Foligno, comm. Canon, I, 1, 4, 2, f. 53rb : « Considerandum autem quod istum ordinem naturalium virtutum obedientium animalibus virtutibus cognitivis omnes philosophi posuerunt et Avicenna voluit hoc specialiter 8 De animalibus capitulo ultimo, cum loquitur de gallina pugnantem contra gallum que fugatur et gallina credidit se esse gallum ; unde dicit sic in hoc percipi potest obedientia naturae cogitationis anime et 3 Canon fen prima capitulum de amore. » 
médicaments, la diète ou les passions (accidentia animae). Pour sa part, Gentile ne commente que la dernière option, en affirmant une fois de plus qu'il n'y a pas nécessairement de corruption de l'humeur au départ de cette maladie. C'est sur la pensée qu'il faut agir. C'est justement ce que la poésie peut faire, comme l'indiquent les symptômes mêmes de la maladie.

Et en particulier : la disposition à la tristesse est altérée lorsqu'il se rappelle la séparation - bien sûr dans les chansons - et l'éloignement, c'est-à-dire la distance qui le sépare de la femme qu'il aime. Avicenne affirme donc que ceux-là, s'ils entendent des chansons d'amour dans lesquelles apparaissent une promesse et l'espoir de la rejoindre, ils rient. $\mathrm{Si}$, en revanche, il s'agit de chansons qui présentent le désespoir et la séparation, ils pleurent ${ }^{110}$.

Les effets des chansons dépendent donc des émotions qu'elles suscitent en évoquant l'amour. Ils dépendent aussi et surtout de la représentation de l'être aimé dans l'esprit. Comme il l'écrit un peu plus loin :

On doit considérer que l'écoute d'une chanson, tantôt rappelle à l'esprit [du malade] la forme de l'amante, tantôt l'en détourne, en raison d'un grand plaisir. Si, en effet, elle l'en détourne, alors elle soigne. Si, au contraire, elle lui rappelle, alors la passion amoureuse augmente. Et ainsi, soit elle la rappelle sous la forme du désespoir, ce qui le fait pleurer ; soit sous la forme de possibles retrouvailles, ce qui le fait rire. Ce sont là des choses contingentes, qui dépendent des dispositions particulières des hommes et de la diversité des chansons et de leur rapport avec ces hommes. [...] Toutes ces choses écartent la pensée du regard porté sur la forme chérie ${ }^{111}$.

Gentile da Foligno insiste donc, plus qu'Avicenne lui-même, sur le rôle de la pensée d'une part, et sur l'importance du langage d'autre part. Nous arrivons ainsi à l'étrange dubitatio qui vient clore notre texte. Dans le Canon, il est simplement écrit que dans ce domaine les mots sont efficaces (verba enim in hoc capitulo conferunt), mais le speculator pense que c'est l'occasion de s'interroger plus précisément sur le pouvoir des mots.

110 Gentile da Foligno, comm. Canon, III, 1, 4, 22, Vat. Lat. 4459, f. 72rb : « Et precipue alteratur dispositio ad tristitiam cum fit rememoratio repudii, scilicet in cantilenis, et elongationis, id est remotionis ad dominam que diligitur. Vult igitur Avicenna quod isti, si audiunt cantilenas amoris in quibus fiat promissio et spes assecutionis, ipsi rident. Si autem sint cantilene in quibus inducatur disperatio et repudiatio, ipsi plorant. »

111 Ibid., f. 72va : «Considerandum quod auditus cantilene vel reducit in mentem formam domine vel propter multam delectationem abstrahit. Siquidem abstrahit, tunc curat. Si vero reducit, [in marg. Et tunc auget ylissci. Et tunc] aut reducit sub forma desperationis, et tunc ipse plorat ; aut sub forma assecutionis possibilis, et tunc ipse ridet. Ipsa autem sunt contingentia secundum dispositiones particulares hominum et secundum diversitates cantilenarum et proportionem earum ad homines. [...] Omnia ista divertunt cogitationem ab aspectu forme dilecte. » 


\section{Le pouvoir de la parole}

La formulation de cette dubitatio ressemble beaucoup à celle de la differentia 156 du Conciliator de Pietro d'Abano ${ }^{112}$. Au début, il s'agit même d'une simple reprise, légèrement aménagée et augmentée, des propos du médecin padouan ${ }^{113}$, et le lien avec la problématique amoureuse apparaît seulement de manière fragmentée, ici ou là, dans quelques arguments faisant appel au pouvoir des images et des émotions. Car, comme l'a montré Vittoria Perrone-Compagni ${ }^{114}$, la thèse de Pietro d'Abano est la suivante : dans le domaine médical, les incantations sont efficaces par l'intermédiaire de la confiance et de l'espoir qu'elles font naître chez le malade, en agissant notamment sur son imagination et ses émotions. Dans cette première partie du texte, qui date de la première rédaction du commentaire au Canon, Gentile da Foligno semble s'accorder avec son aîné. Aussi retrouvet-on, parmi les arguments invoqués pour défendre le pouvoir des mots et des incantations sur l'âme et sur le corps des malades, une citation du De anima d'Avicenne sur l'efficacité de la confiance dans la guérison des malades et une autre du De motu animalium d'Aristote, où il est dit que les formes (species) dans l'imagination agissent sur le corps ${ }^{115}$. Une première solution, qui n'est autre que celle de Pietro d'Abano, voit donc le jour : les incantations, en tant que telles, ne participent pas à la guérison ${ }^{116}$. Autrement dit, ce ne sont pas les mots prononcés, c'est-à-dire les sons, entités physiques émises dans l'air, qui sont efficaces par

112 Pour le texte de Pietro d'Abano, cf. B. Delaurenti, " Pietro d'Abano et les incantations. Présentation, édition et traduction de la differentia 156 du Conciliator », dans J. -P. Boudet, N. Weill-Parot (éds.), Médecine, astrologie et magie entre Moyen Âge et Renaissance : autour de Pietro d'Abano, Florence 2012, 39-105.

113 Pour une comparaison précise entre la differentia 156 et la quaestio de Gentile (dans la version conservée dans les Quaestiones extravagantes), voir Delaurenti, La puissance des mots, 40-41, 45-46, 51-56, 75-76, 84-87 (et passim).

114 V. Perrone-Compagni, « La differenza 156 del Conciliator : une rilettura », Annali del Dipartimento di Filosofia (Firenze) 15 (2009), 65-107.

115 Gentile da Foligno, comm. Canon, III, 1, 4, 22, Vat. Lat. 4459, f. 72va-vb : « Item, non minoris est entitatis incantatio et similia quam species in anima ; sed tales species alterant, ut Aristoteles in libro De causa motus animalium, ubi dicit alterant autem sensus et fantasie ; ergo etc. Amplius, illud potest curare quod potest vehementiam spei sanitatis inducere ; sed incantatio et similia hoc possunt ; ut est notum. Maior probatur, quia spes de sanitate quandoque plus facit quam medicus cum instrumentis, [f. $72 \mathrm{vb}$ ] ut Avicenna 6 De naturalibus, particula $4^{\mathrm{a}}$, penultimo capitulo ; et ideo ille plures sanat de quo plures confidunt, ut primo Pronosticorum $2^{\circ}$ commento. » 116 Ibid., f. 72vb : «In hac questione est primo modus dicendi Conciliatoris. Et ponit diffinitionem precantationis, dicens quod precantatio est oratio admiranda affectione explicata in subsidium precantati, et precipue confidentis. Et post multa verba, vult quod precantatio inquantum precantatio nihil confert ad curam. » 
leurs propriétés intrinsèques. Si tel était le cas, alors quiconque prononcerait certaines paroles obtiendrait les mêmes effets, dans n'importe quelle circonstance et sur n'importe quel individu. Il en va autrement, selon notre médecin, et c'est pourquoi il faut concéder que les paroles n'agissent qu'en fonction de ce que le locuteur place en elles et de ce qu'elles provoquent en nous. C'est seulement indirectement, à travers les images véhiculées dans le discours, et les émotions que celles-ci engendrent dans l'esprit, que les mots agissent. À un premier niveau, donc, on comprend que la poésie puisse être efficace à la manière des incantations du médecin.

Est-ce la seule origine possible du pouvoir des mots ? Non, nous dit Gentile da Foligno, qui reprend à son compte la longue série d'exemples de paroles efficaces fournie par Pietro d'Abano : les sacrements, l'ars notoria, les formules divinatoires, les paroles prononcées aux oreilles des chevaux, etc. Les causes de l'efficacité de ces actes de parole ne sont pas les mêmes selon les cas $^{117}$. Le pouvoir des mots est tantôt lié à la noblesse de l'âme, comme le suggérait Avicenne, tantôt il est seulement l'effet de l'espoir ou de la confiance induite par la parole, parfois c'est Dieu qui agit, parfois ce sont des anges, bons ou mauvais, parfois ce sont les configurations astrales, et parfois l'effet se produit simplement par accident. Jusqu'ici, les scribes du XV siècle et les éditeurs de la Renaissance ne se sont pas trompés : le texte ressemble à s'y méprendre à celui de Pietro d’Abano. La suite du texte, en revanche, ajoutée quelques années plus tard, ne se trouve pas chez le médecin de Padoue et contient des considérations originales.

Gentile da Foligno y continue son argumentation en distinguant deux manières d'envisager la question du pouvoir de la parole, celle des aristotéliciens et celle des platoniciens. Selon la première voie, empruntée par plusieurs médecins médiévaux, depuis Urso de Salerne, à la fin du XII ${ }^{\mathrm{e}}$ siècle ${ }^{118}$, jusqu'à Iacopo da Forli au tournant du XV $\mathrm{XV}^{\mathrm{e}}$ siècle $\mathrm{e}^{119}$, la parole agit sur le corps par l'intermédiaire du pouvoir de l'imagination.

Si l'on considère les propos d'Aristote et de ses disciples, lesquels se sont tous efforcés de reconduire les effets naturels à leurs causes naturelles, on pourra seulement tirer d'eux que si les incantations et les amulettes font quelque chose, c'est parce qu'elles produisent une imagination forte et fixe, ces imaginations ou fantasmes dont ils ont affirmé qu'ils ont le

117 Gentile da Foligno, comm. Canon, III, 1, 4, 22, Vat. Lat. 4459, f. 72vb : « Cum ergo huiusmodi incantationes sive verba videantur inducere effectus reales videnda est causa. »

118 Sur Urso de Salerne, voir M. Van der Lugt, « The Learned Physician as a Charismatic Healer : Urso of Salerno (Flourished End of Twelfth Century) on Incantations in Medicine, Magic and Religion », Bulletin of the History of Medicine 87/3 (2013), 307-346.

119 Robert, « Le pouvoir des incantations ». 
pouvoir de nous transformer, comme cela est résumé par Aristote dans le traité Sur le mouvement des animaux et dans la particule $7 \mathrm{du} 8^{\text {ème }}$ problème des Problèmes ${ }^{120}$.

Le véritable aristotélicien est donc naturaliste, c'est-à-dire exclusivement naturaliste : il cherche partout des causes naturelles aux effets constatés, et refuse de faire appel aux esprits ou autres démons. Il ne peut en être autrement si l'on se conforme à la description péripatéticienne de l'univers, qui ne conçoit d'autre substance séparée que Dieu, les intelligences qui meuvent le ciel et, peut-être, l'âme intellective humaine ${ }^{121}$. Que faire, dans ces conditions, des témoignages qui racontent l'intervention des esprits ou des démons sur eux-mêmes ? Gentile da Foligno raconte une anecdote à ce propos. Un de ses amis, un certain Giovanni di Ascognano, vit en plein jour un militaire noir marcher près de lui dans les rues de la ville, alors qu'à l'évidence il n'y avait personne. Gentile précise qu'il fut immédiatement pris d'un vertige mélancolique ${ }^{122}$. Les illusions de ce genre sont encore plus fréquentes la nuit, nous dit Gentile. Certains croient voir dans les arbres en mouvement ou dans leurs ombres des géants armés s'avançant vers eux ${ }^{123}$. Dans tous ces cas, c'est l'imagination qui vient renforcer une perception indistincte. En ce qui concerne les démons, l'illusion n'est pas nécessairement sensible, elle peut être directement causée par l'imagination. Dans les cas où les démons ne sont que des fantasmes et lorsque, par ailleurs, on peut expliquer le pouvoir de la parole par cette même imagination qui nous plonge dans l'illusion, alors il n'est point besoin d'aller chercher d'autres causes que les images et/ou les humeurs.

120 Gentile da Foligno, comm. Canon, III, 1, 4, 22, Vat. Lat. 4459, f. 72vb : « Si quis autem considerat dicta Aristotelis et suorum sequacium qui omnes effectus naturales conati sunt in suas causas naturales reducere, solum ab eis haberi poterit, quod si incantationes et colli suspensiones faciunt aliquid, est quia faciunt aliquam fortem et fixam ymaginationem, quas imaginationes sive fantasias posuerunt habere vim transmutativam in nobis, sicut ab Aristotele sumitur in libro De causa motus animalium et $7^{\mathrm{a}}$ particula de Problematibus $8^{\circ}$ problemate. »

121 Gentile da Foligno, comm. Canon, III, 1, 4, 22, Vat. Lat. 4459, f. 72vb : «Quod autem iste incantationes valeant propter demones non est conforme dictis peripateticorum, qui non posuerunt alias substantias separatas nisi deum, intelligentias moventes orbem et animal intellectivam humanam, ut apparet ex 12 Metaphysice et $3^{\circ}$ De anima. »

122 Ibid. : « Illud autem quod homines dicunt se vidisse diabolos vel angelos, ipsi dicunt esse delusiones circa sensus que contingunt vel quia species ab intra revertuntur usque ad particulares sensus, verbi gratia ad visum, et iudicatur aliquid nigrum vel album vel animal vel aliud extra esse, cum tamen non sit, [in marg. ut $3^{\circ}$ Colliget], sicut accidit cuidam meo amico domino Johanni de Ascugnano, qui veniens in die de villa, videbat semper ante se, precedere unum militem nigrum, et tamen nichil precedebat, ut famulus dixit. Et tandem incidit vertiginem melancolicam. » 123 Ibid. : « Accidit etiam talis delusio de nocte incedentibus propter non bene distinguere vel quia diversimode presententur rerum imaginationes, unde quandoque arbores credunt esse homines armatos vel homines valde magnos. Et sicut hoc accidit circa visum, ita circa alios sensus. » 
Cette analyse naturaliste des apparitions démoniaques ressemble fort à celle proposée par Witelo, le grand spécialiste de l'optique, qui étudia à Padoue et fut actif à la cour pontificale de Viterbe à la fin du XIII ${ }^{\mathrm{e}}$ siècle. Dans son Epistula de natura daemonum, rédigée vers 1268, il affirmait en effet que la plupart du temps les apparitions démoniaques ne sont que des illusions, des formae imaginata $^{124}$. Parmi les exemples d'illusions, Witelo mentionne celle liée à l'amor hereos, lorsque l'amant voit sa bien-aimée alors qu'elle est n'est pas là - par le simple pouvoir de l'imagination - et pense qu'il subit une illusion démoniaque ${ }^{125}$. On trouve aussi un propos semblable dans le De improbatione maleficiorum du médecin Arnaud de Villeneuve : lorsqu'une personne pense être soumise à une influence démoniaque, elle souffre en fait, la plupart du temps, de mélancolie ${ }^{126}$. Pour illustrer son propos, le médecin se contente de renvoyer à son traité $D e$ amore heroico $^{127}$. Pour l'aristotélicien, la passion amoureuse n'est qu'un de ces effets de l'imagination, pareil aux illusions dont nous sommes victimes lorsque le sensible nous trompe et que l'imagination surenchérit, déformant un peu plus encore ce qui n'était au départ qu'une perception vague.

L'approche des platoniciens est totalement différente et radicalement opposée à celle des aristotéliciens, puisqu'elle consiste à accepter pleinement l'existence des esprits et des démons, ainsi que leur intervention dans le cours de la nature, et à admettre en outre la possibilité, pour nous, de solliciter leur action par des incantations. Cette théorie, écrit Gentile da Foligno, « plus conforme à mon intellect, est qu'il existe dans notre espace une multitude indénombrable de

$124 \mathrm{Il}$ convient toutefois de noter que Witelo accepte l'existence des démons et leur interaction avec les humains. C'est notamment le cas des incubes et des succubes qui viennent s'accoupler avec les êtres humains. Sur ce point, voir M. Van der Lugt, Le ver, le démon et la vierge. Les théories médiévales de la génération extraordinaire, Paris 2004, 296-302.

125 Witelo, Epistula de daemonibus, dans J. Burchardt (éd.), List Witelona do Ludwika we lwówku Slaskim : Problematyka Teoriopoznawcza Kosmologiczna i Medyczna, Wroclaw 1979, 170 : « Hominibus etiam vigilantibus saepe videntur mirabilia. Et hoc fit per fortem imaginationem, formam suam sensui communi imprimentem, ut patet in<a>moratis amore hereos, qui formam sensibilem amati primo debiliter phantasiae impresserunt, quam cum saepe replicaverunt apud illam, conservaverunt diutius. [...] Tunc itaque tales inamorati se credunt amata praesentialiter videre. Et cum apprehendere putant, surgunt aliqualiter propter motus spiritus in capite isporum, et tandem, cum spiritus redeunt ad posterius capitis, formae visae evanescunt. Et dicunt se illi per daemones illusos. "

126 Arnaud de Villeneuve, Epistola de reprobacione nigromantice ficcionis (de improbatione maleficiorum), éd. S. Giralt, dans Arnaldi de Villanova Opera medica omnia, VII, 1, Barcelona 2005, passim (notamment 232-233).

127 Ibid. 
ces démons et qu'ils sont des substances éternelles existant par elles-mêmes ${ }^{128}$. Ce n'est pas une simple croyance, explique Gentile, puisque de nombreux philosophes extrêmement sages (philosophi sapientissimi) ont défendu cette thèse depuis Platon et que les multiples attaques contre elle n'ont jamais affaibli sa présence dans l'histoire. Gentile ajoute cependant un argument supplémentaire : nous serions parfois contraints de postuler l'existence de ces entités spirituelles lorsqu'aucune autre cause naturelle ne peut être avancée pour expliquer des phénomènes extraordinaires. Il donne comme exemple le cas d'un homme devenu soudainement poète, tiré du Problème XXX, $1 \mathrm{du}$ pseudo-Aristote ${ }^{129}$. Selon le point de vue aristotélicien, la cause d'un tel phénomène doit se trouver dans l'humeur mélancolique ; une cause naturelle donc, et qui plus est corporelle. Ni Dieu, ni démon. Or, poursuit Gentile da Foligno, une telle explication repose sur un jeu d'analogies trompeuses et extravagantes entre la bile noire et le vin. De plus, ajoute-t-il, si les humeurs pouvaient à elles seules expliquer de telles transformations dans les puissances de l'âme, en l'occurrence la connaissance et le langage, pourquoi n'observe-t-on jamais la naissance de bébés savants ? Ne pourrait-on imaginer, de manière contrefactuelle, un excès naturel de bile noire à la naissance qui engendrerait un tel génie ? Se servant d'Averroès contre Aristote, il montre que l'acquisition d'un talent ou d'un savoir ne saurait dépendre uniquement d'un changement d'humeur. Les propositions et même les images de la fantasia du sage ou du poète viennent toujours d'un maître ou d'un livre. Si le génie poétique intervient soudainement dans la vie d'un individu, indépendamment de toute éducation et de tout entraînement, ce ne peut donc être le fait

128 Gentile da Foligno, comm. Canon, III, 1, 4, 22, Vat. Lat. 4459, f. 72vb : « Illud autem quod meo intellectui est conformius est, quod in nostro (sap. del.) spatio, sit multitudo fere innumerabilis horum demonum et quod sint substantiae quedam eterne de se. »

129 Ibid., f. 72vb-73ra : « Et una ratio [f. 73ra] : nam videmus effectus non reducibiles in alias causas, de quibus sunt, quod inlitteratus subito fiat litteratus ; unde famosum est quosdam (sic pro quod ?) et quasdam (sic pro quidam ?) que prius non cognoverant litteras modo subito loqui litteraliter. Et Aristoteles ipse dicit in libro de Problematibus $30^{\mathrm{a}}$ particula primi problemate, quod homo quidam, nomine Maracus, non erat poeta ; quando excedit, id est quando factus est melancolicus, et videtur sentire Aristoteles, quod tunc factus est poeta. Narrat etiam Guilelmus Lombardus simile huic. Et multi talia non possent habere causam alteram nisi spiritus istos. Aristoteles autem vult hoc attribuere humori melancolico, sed si quis actendit causae, que ab Aristotele dantur, sunt valde longinque ; imaginatur enim quod melancolia assimilatur vino, et sicut vinum varia facit accidere, sic et melancolicus humor. Tamen si melancolicus humor potest corpus sic disponere, et hoc quando, secundum naturam, dominatur corpori, mirabile est quod aliquis non nascitur talis (quia del.) qui omnem linguam et omnem scientiam vel aliquam habeat a seipso sine aliquo libro vel magistro. » 
d'une cause corporelle. Gentile pense qu'il n'y a d'autre choix que d'accepter dans ce cas l'intervention d'un esprit.

Le texte se poursuit par une longue défense métaphysique et cosmologique de l'existence des esprits et des démons, essentiellement fondée sur $L e$ démon de Socrate d'Apulée et le commentaire de Calcidius au Timée de Platon ${ }^{130}$. Gentile décrit alors à grands traits les principes de l'organisation harmonique du monde platonicien, et va jusqu'à rappeler l'analogie proposée par Calcidius entre démons et nombres ${ }^{131}$. Il convient de noter que ces références ne sont pas rares dans l'œuvre de Gentile da Foligno. Dans les prologues des livres I et III $\mathrm{du}$ Canon, par exemple, il tente indique qu'il faut invoquer la perfection divine en médecine, en considérant Dieu comme une forme de démiurge, à la manière de Galien et Platon. Il fonde alors son discours sur les autorités médicales en particulier Galien et son De utilitate partium - mais aussi sur le Timée de Platon, la Métaphysique d'Avicenne, celle d'al-Ghazzali, ou encore les Lettres de Sénèque ${ }^{132}$. Ailleurs, dans ses sermons universitaires prononcés à Pérouse dans les années 1340, si Sénèque est l'auteur le plus cité (50 fois), Gentile prend à deux reprises des citations de Macrobe et d'Apulée comme thèmes, et l'on trouve dans ces textes des références fréquentes à Platon ( 6 fois), Apulée (13 fois) et même à Proclus (8 fois $)^{133}$. Notre texte illustre donc cette culture platonicienne. Dans le contexte qui nous intéresse, il s'agit surtout de se déprendre de l'empirisme des aristotéliciens et de se laisser convaincre, faute de preuves tangibles, par la thèse platonicienne, car, « si l'argument ne démontre pas, il est cependant

130 Ibid., f. 72vb-73ra : « Sed qui sequitur doctrinam Platonis quam ponit Lucius Apulegius in libro De deo Socratis, habet ponere hos demones cohabitantes sive conversantes nobiscum. [...] Et hec est ratio que ab antiquis inducebatur quando volebant probare hos demones esse. Et est ratio que imponitur Calcidio in commento Tymei. » Dans le chapitre sur la mélancolie, on trouve la même référence à Platon et Apulée. Cf. Comm. Canon, III, 1, 4, 18, Venise 1520, f. 86ra : « Considerandum circa primam partem quod Azaravius capitulo de epilepsia ponit hoc quod Avicenna dicit : multa enim videmus mirabilia non reducibilia in causa naturales nobis notas. Quidam enim melancolici illiterati loquuntur literaliter, ut Gulielmus ponit. Quidam prophetizant. Utrum autem hoc possit fieri a melancolico humore sive demonio, ut Aristoteles sentit 30 particula de Problematibus problemum 1, habet dubium. Tamen multi philosophi reducunt impressionem huiusmodi in demonia, sicut Azaravius, et est conforme sententie Platonis et scientie Apullegii in libro De deo socratis. » En fait, Azaravius (ou Albucasis) ne dit pas vraiment cela et mentionne simplement l'opinion de ceux qui font appel aux démons. Cf. Albucasis, Liber theoricae necnon practicae, Augsburg 1519, f. 33rb.

131 Sur cette comparaison, voir J. Den Boeft, Calcidius on Demons, Leiden 1977.

132 Gentile da Foligno, comm. Canon, I, prologus, éd.cit., f. 2ra-b ; et III, prologus, t. 3, f. 1ra-b. 133 Voir C. Schlam, « Graduation speeches of Gentile da Foligno », Mediaeval Studies 40 (1978), 96-119. Voir aussi J. Agrimi et C. Crisciani, Edocere medicos. Medicina scolastica nei secoli XIIIXV, Napoli 1998, 257-261. 
suffisamment persuasif ${ }^{134}$. La fin du texte montre tout de même une certaine prudence : notre auteur écrit que si ses propos paraissent incompatibles avec la foi, qu'on les oublie (si autem hec dicta non sunt consona veritati nostre fidei habeantur pro non dictis).

Gentile da Foligno se contente peut-être simplement ici de répondre à cette dubitatio sur le pouvoir des incantations en partant de la présentation de Pietro d'Abano, pour mieux la dépasser ensuite, en montrant que, dans certains cas au moins, il faut admettre que nos paroles suscitent l'action des démons pour produire des effets, ce que l'auteur du Conciliator ne développait pas ${ }^{135}$. Mais pourquoi avoir introduit un tel excursus à cet endroit précis, après le commentaire du chapitre du Canon consacré à l'amour ? À un premier niveau, il s'agit de savoir comment la parole peut améliorer ou dégrader l'état du malade. De ce point de vue, la solution apportée par Pietro d'Abano, c'est-à-dire celle des aristotéliciens, paraît suffisante. La parole agit sur les sens, puis sur l'imagination, et produit des effets, soit par l'image elle-même, soit par les émotions engendrées par le miroir déformant de la faculté estimative. Pourquoi terminer ce texte en clamant haut et fort son allégeance à Platon? Au vu des cas discutés par Gentile da Foligno l'illusion et le génie mélancolique - et des sources qu'il utilise - Apulée et Calcidius - ne peut-on imaginer qu'il revienne, par des voies détournées, sur la question de l'origine divine de l'amour évoquée dans le commentaire littéral de ce chapitre du Canon d'Avicenne?

Dans un premier temps, après avoir rappelé la définition platonicienne de la passion amoureuse, notre médecin s'était contenté de limiter le rôle des causes physiques de cette mélancolie d'un genre particulier, tout en refusant, apparemment, l'origine strictement divine de cette passion. Tout se passe d'abord dans l'âme, et plus particulièrement dans les images. La critique du Problème $\mathrm{XXX}, 1$, dans cette partie ultérieure du texte, montre que sa position est plus radicale encore. Le genre de fureur poétique décrite par le pseudo-Aristote provient d'un esprit et non d'un excès d'humeur. Si le Problème XXX, 1, avait pour but de donner une explication physico-médicale aux thèses de Platon, nous assistons ici, à travers la critique de Gentile da Foligno, à un retour du platonisme.

Le propos de Gentile da Foligno ne rappelle d'ailleurs pas tant les thèses du Phèdre, avec ses distinctions sur les différentes formes de folie divine, que celles

134 Gentile da Foligno, comm. Canon, III, 1, 4, 22, Vat. Lat. 4459, f. 73(bis)v : « Nos autem dicamus quod ratio etsi non demonstret, tamen satis suadet. »

135 Ibid. : «Utrum autem isti demones moveantur incantationibus et caracteribus est res de qua nihil dico, cum famosum est quod sic. Et si hoc est, incantationes valent et verba et consimilia. Ex quibus solvitur questio et conceduntur omnia argumenta que incantationes agant, si demones nostris verbis et karacteribus moventur. » 
$\mathrm{du}$ Banquet, en particulier lorsque Diotime corrige Socrate : Eros n'est pas un grand dieu, c'est un esprit, à mi-chemin entre les dieux et les mortels (202d-e). Si, dans le Phèdre, Eros est à l'origine de la folie amoureuse, et les muses sont causes de la folie poétique, dans le Banquet il semble que toutes ces fureurs se produisent par l'intermédiaire d'un daimon. Or, le Banquet est une référence explicite du Démon de Socrate, et Apulée rappelle explicitement que les démons les plus nobles sont Sommeil et Amour ${ }^{136}$. De plus, Gentile da Foligno connaît les Métamorphoses, qu'il cite dans un autre passage de son commentaire au Canon, et connaît donc peut-être le mythe d'Amour et Psychè ${ }^{137}$. La lecture de ce texte laisse donc une impression étrange, comme si la passion amoureuse était en fin de compte la même que celle du poète, ni purement corporelle, ni divine, mais soumise à l'inspiration d'un esprit supérieur.

Selon Giorgio Agamben, c'est précisément ce passage par la démonologie d’Apulée et de Calcidius qui, en transmettant celle de Platon, aurait participé à la construction d'une vision « basse » de l'amour, en identifiant Eros aux démons aériens, mais aussi aux héros, d'où, selon lui, l'expression amor heroicus. Comme il l'écrit :

C’est cette trinité sémantique Eros-héros-démon aérien qui, en se fondant dans une antique théorie médicale dont on trouve la trace dès Plutarque et Apulée et selon laquelle l'amour est une maladie, donne naissance à l'image 'démonique' et sinistre d'un Eros déjà décrit par Plutarque, hors de toute influence chrétienne, comme un petit monstre pourvu de crocs et de griffes. Dans le cadre de la tradition néo-platonicienne s'était donc élaborée la représentation 'basse' d'un Eros-héros-aérien, occupé à piéger les hommes en leur inspirant de folles passions ; et c'est cette représentation, en liaison avec la vieille idée hippocratique du héros fauteur de maladie mentale, qui a probablement donné lieu, sinon à la formule médicale traditionnelle de l'amor hereos, du moins à son interprétation comme amor heroycus, 'amour héroïque'138.

Giorgio Agamben ne mentionne aucun texte du Moyen Âge pouvant étayer cette interprétation, passant directement d'Apulée et Calcidius aux « fureurs héroïques » de Giordano Bruno. C'est sans doute que le texte de Gentile da Foligno est l'un des rares à permettre de tels rapprochements. À notre connaissance, Mary Frances Wack est la seule à avoir repéré dans ce commentaire de Gentile da Foligno un tournant historique dans les théories médicales de la passion amoureuse, précisément parce qu'il fait intervenir des considérations magiques, ce qui deviendrait

136 Apulée, Le démon de Socrate, trad. C. Lazam, Paris 1993, 64-65.

137 Gentile da Foligno, comm. Canon, III, 1, 5, 8, f. 98ra.

138 Agamben, Stanze, 199-200. 
de plus en plus fréquent au siècle suivant, en tout cas à partir de Marsile Ficin ${ }^{139}$. Malheureusement, ne connaissant que la version transmise par les éditions de la Renaissance, elle n'eut pas accès à la partie sur les démons. C’est pourquoi elle écrivait : "Gentile conclut, avec un scepticisme typique de la philosophie naturelle (et donc de la médecine qui en est une sous-espèce) du XIV siècle, que 'ni l'homme, ni Dieu, ne peuvent faire quoi que ce soit à travers les incantations' " $\mathrm{Au}$ contraire, comme Pietro d'Abano, il considère que les mots agissent parfois par les images et les émotions produites chez l'auditeur, mais parfois aussi grâce à l'intervention d'esprits et autres démons. La forme particulière de platonisme défendue ici par Gentile da Foligno pourrait donc préfigurer certaines thèses défendues par Marsile Ficin, près d'un siècle plus tard, dans son commentaire au Banquet de Platon.

\section{Conclusion}

Nous espérons avoir montré l'originalité du commentaire de Gentile da Foligno sur le chapitre consacré à l'amour dans le Canon d'Avicenne par rapport à la tradition médicale antérieure. Ajoutons qu'il est aussi original relativement à certains témoignages ultérieurs, comme le commentaire de Jacques Despars sur ce même chapitre, écrit près d'un siècle plus tard. Le médecin parisien y reprend à son compte la vieille étymologie de l'amor hereos, faisant de la passion amoureuse une maladie masculine qui touche essentiellement les nobles, et insiste surtout sur le rôle de l'humeur mélancolique et sur les causes corporelles de cette maladie. Rien sur le langage, rien sur la poésie, rien sur les démons. Hapax inclassable, ce texte de Gentile da Foligno avait en partie échappé à la critique. Ainsi, Raymond Klibansky écrivait : « Il fallut attendre la XV siècle pour que l'on assimilât expressément la mélancolie aristotélicienne à la 'fureur divine' de Platon. C'est alors - et alors seulement - que l'époque moderne conçut la notion moderne de génie, réac-

139 M. -F. Wack, « From Mental Faculties to Magical Philters : The Entry of Magic into Academic Medical Writings on Lovesickness, 13th-17th Centuries », dans D. A. Beecher et M. Ciavolella (éds.), Eros and Anteros. The Medical Traditions of Love in the Renaissance, Ottawa 1992, 9-31. Elle écrit $(15-16):$ : [...] it was not until the fourteenth century that the debates on imagination and magic were perceived as rlevant to the medical doctrines on lovesickness. The crucial step of linking visual species and the imagination to magic in the particular context of lovesickness is well illustrated in Gentile da Foligno's mid-fourteenth century commentary on Avicenna's Canon. In the chapter on ilisci or lovesickness, Gentile's interest in natural magic prompts him to ask whether incantations and suspensions are of any use in curing the lover's malady. »

140 Ibid. 
tivant, certes, d'antiques notions, mais en les investissant d'un sens nouveau ${ }^{\mathbf{1 4 1}}$. La singularité de notre texte, dans l'Italie des années 1330-1340, tient en effet à la relation inédite qu'il parvient à établir entre passion amoureuse et poésie, en déterminant précisément le type de mélancolie dont souffrent les amants, mélancolie qui a finalement peu en commun avec celle de la tradition aristotélicienne, expliquée uniquement par la bile noire. Véritable maladie de l'âme, de l'imagination, mais aussi du jugement et surtout du désir, les atteintes corporelles viennent après. Dans un second temps seulement le mal corporel augmente les souffrances de l'âme, plaçant l'individu dans le cercle des actions réciproques de l'âme et du corps. Ce qui caractérise l'homme souffrant de cette passion, c'est son détachement, son repli dans l'imagination. Comme le poète génial, son imagination est inspirée, par quelque chose de supérieur. Gentile da Foligno serait donc l'un des premiers, au Moyen Âge, à utiliser Platon pour parvenir à une description de la souffrance amoureuse qui conviendrait aussi à celle du poète génial.

Pour toutes les raisons évoquées plus haut - mention faite de son expérience poétique, discussion du génie et de l'inspiration du poète, dimension mentale de la passion, intérêt pour le pouvoir de la parole - il nous a donc paru utile d'inscrire le commentaire de Gentile da Foligno dans le contexte plus large des débats poétiques qui agitaient ses contemporains. Lu sous cet angle, notre texte paraît donner des armes théoriques pour les débats poétiques de l'époque. Dans le même temps, il semble aussi rappeler qu'il existe un espace propre à la poésie, qui n'est pas totalement réductible au discours savant du médecin. C'est pourquoi il réserve le traitement de certaines questions, pourtant techniques, à ses poèmes, comme si le discours médical s’arrêtait parfois là où commence celui du poète. Le philosophe Jean de Jandun semble dire la même chose dans son commentaire au livre VII de la Physique d'Aristote. Alors qu'il discute de la possibilité d'une altération du corps par l'imagination, il achève son commentaire en mentionnant l'amor hereos. Pour saisir la nature du phénomène amoureux, explique-t-il, il lui faudrait raconter des histoires. C'est pourquoi il préfère se taire, parce qu'il n'est pas poète ${ }^{142}$. À un certain point donc, le philosophe et le médecin doivent céder

141 Klibansky, Panofsky et Saxl, Saturne et la mélancolie, 399.

142 Jean de Jandun, Quaestiones super libros Physicorum, VI, éd. 1485 (sans lieu d'édition), f. 113ra : "Quod tamen alteratio (sic pro imaginatio) sit causa multarum alterationum in corpore imaginantis, hoc non est impossibile, ut tactum est. Et huiusmodi ratio potest capi ex illis qui vocantur amor chereos (sic pro hereos). Adeo enim fortiter et continue imaginantur rem amatam et concupiscunt, quod ipsi incidunt in egritudines magnas, et cum difficultate sanari possunt nisi habeant quod concupiscunt, quod non contingeret nisi in eorum corporibus alteratio fieret ab imaginatione in actu ; de hoc ego bene posset (sic pro possem ?) narrare aliquas historias de ista materia, sed eas dimitto propter brevitatem et quia non sum poeta. » 
la place aux poètes. Cette reconnaissance d'une autonomie de la poésie explique peut-être le caractère succinct et quelque peu énigmatique du commentaire de Gentile da Foligno, qui s'en tient à ce qu'il peut dire en tant que médecin. En tout cas, ses propres remarques devraient nous inviter à aller chercher dans la poésie elle-même la révolution qui fit naître la figure moderne du poète génial. 
